\title{
Design, Synthesis and Preliminary Pharmacological Screening (antimicrobial, analgesic and anti-inflammatory activity) of Some Novel Quinazoline Derivatives
}

\author{
Biswajit Dash $^{1 *}$, Suvakanta Dash ${ }^{1}$, Damiki Laloo ${ }^{1}$, Chitrani Medhi ${ }^{2}$ \\ ${ }^{1}$ Girijananda Chowdhury Institute of Pharmaceutical Science, Azara, Hathkhowapara, Guwahati-781017, Assam, India. \\ ${ }^{2}$ Department of Chemistry, Gauhati University, Gopinath Bordoloi Nagar, Guwahati-781014, Assam, India.
}

\begin{tabular}{|c|c|}
\hline ARTICLE INFO & ABSTRACT \\
\hline Article history: & Objectives: The present research work is designed to synthesize some new series of quinazoline-4-one/4-thione \\
\hline Received on: $06 / 02 / 2017$ & derivatives by modifying the structures retaining the fundamental structural features for the biological activity \\
\hline Accepted on: 30/03/2017 & and screened for their anti-microbial, analgesic and anti-inflammatory properties. \\
\hline Available online: $30 / 06 / 2017$ & Material and methods: A series of 7-chloro-3-[substituted (amino/phenyl amino)]-2-phenyl quinazolin-4(3H)- \\
\hline \multirow{3}{*}{$\begin{array}{l}\text { Key words: } \\
\text { 7-chloro-2-phenyl-4H- } \\
\text { benzo[d][1,3]oxazin-4-one, } \\
\text { quinazoline derivatives, anti- } \\
\text { microbial activity, analgesic } \\
\text { and anti-inflammatory } \\
\text { activity. }\end{array}$} & $\begin{array}{l}\text { one/thione derivatives and } 1-(7-c h l o r o-4-0 x O /-2-\text { phenylquinazoline-3-(4H-yl }) \text {-substituted urea derivatives were } \\
\text { prepared and characterized from different spectra and elemental analysis. The anti-microbial, analgesic and anti- } \\
\text { inflammatory activity were investigated by agar diffusion cup plate method, tail immersion method and } \\
\text { carrageenan-induced paw oedema method respectively. }\end{array}$ \\
\hline & $\begin{array}{l}\text { Results: The physico-chemical and spectroscopic data confirmed the synthesis of quinazoline derivatives. Five } \\
\text { compounds (IIc, IIg, IIh, IIi and IIj) showed good activity against microbes and two compounds (IIc and IIg) } \\
\text { showed good activity profile against both pain and inflammation. Three compounds (IIh, IIi and IIj) shows } \\
\text { good therapeutic activity only against inflammation. }\end{array}$ \\
\hline & $\begin{array}{l}\text { Conclusion: The quinazoline derivatives obtained indicates that the methyl/methoxy group in phenyl hydrazine } \\
\text { ring, amine, urea and thiourea substitution at } 3^{\text {rd }} \text { position of quinzoline ring are essential for anti-microbial, } \\
\text { analgesic and anti-inflammatory activity. Compounds IIc, IIg, IIh, IIi and IIj were found to be potent which } \\
\text { may be effective as potential source for the development of anti-microbial, analgesic and anti-inflammatory } \\
\text { compound. }\end{array}$ \\
\hline
\end{tabular}

\section{INTRODUCTION}

Microbial infections cause pain and inflammation in the body. Generally two groups of agents are given for normal practice simultaneously (anti-microbial, analgesic and antiinflammatory effect). Compound with all three properties are not very common. The commercially available antimicrobial agents are having many adverse effects (Alagarsamy et al., 2005)

\footnotetext{
* Corresponding Author

Biswajit Dash, Girijananda Chowdhury Institute of Pharmaceutical Science, Azara, Hathkhowapara, Guwahati-781017, Assam, India. Email: biswajit.itbhu@gmail.com
}

and increased risk of antibiotic ( $\beta$-lactam antibiotic like penicillin and broad spectrum antibiotic like tetracycline) resistance among pathogenic bacteria has become a serious problem for the clinical management of infectious diseases and hence the treatment of bacterial infections remains a challenging therapeutic problem (Lokhandwala and Patel, 2013; Chavan et al., 2014; Sarvanan et al., 2015). Pain is associated with inflammation. Inflammation is a biological response to a series of biochemical reactions whose major function is to protect the body from infections and tissue damage due to injury.

But it is an ill defined, unpleasant feeling caused by nociceptive agents. In the last few decades, first generation non- 
steroidal anti-inflammatory drugs (NSAIDs) had become well established in the treatment of pain and inflammation. Research was going on in these areas which lead to the discovery of second and third generation NSAIDs. From that study, some quinazoline derivative drugs are evolved as third generation NSAIDs such as proquazone, fluproquazone etc. It has been found that the pharmacological profile (anti-inflammatory response) of proquazone is quiet remarkable as compared to indomethacin (Chandrika et al., 2008). But the chronic uses of NSAIDs cause various adverse effects such as bleeding, gastro-intestinal lesion and nephrotoxicity as revealed through literature (Hemlatha and Girija, 2011).

The application of heteroaromatic compounds in the field of medicinal chemistry has been recognized and has been studied experimentally due to potent pharmacological activity. Heterocyclic compounds containing nitrogen performs a broad spectrum of biological activity (El-Gazzar et al., 2009). Hence the search of our interest is based on the undiscovered synthetically accessible heterocyclic template which is capable of bearing potential pharmacophoric group to elicit and enhance pharmacological activity such as chemotherapeutic, analgesic and anti-inflammatory principle.

In the recent years, the chemistry of quinazoline and its derivatives has received considerable attention owing to their synthetic and effective biological importance. The connection between a wide spectrum of biological activities and compounds containing quinazoline moiety has been known and is well documented in the literature. So with proven pharmacological significance, quinazoline scaffold has taken wide attention for the investigators and their efforts were quite significant as mentioned in the literature and it clearly demonstrate the remarkable potential of quinazoline derivatives as source of useful pharmacophore for the new drug discovery.

In the literature, it has been reported that the quinazoline and its derivatives possesses a wide range of biological activities such as anti-convulsant (Adel S et al., 2012; Srivastava and Kumar, 2004; Jatav et al., 2008; Sarvanan et al., 2012), Central nervous system (CNS) depressant (Kashaw et al., 2009; Jatav et al., 2008; Kashaw et al., 2008), anti-cancer (Adel et al., 2010), anti-tubercular (Mosaad et al., 2004) and anti-malarial activity (Jiang et al., 2005). By analyzing the structural activity relationship (SAR) of synthesized quinazoline derivatives from literature survey, it revealed that substitution of different heterocyclic moieties at $2^{\text {nd }}$ or $3^{\text {rd }}$ position of quinazoline nucleus alters the biological activity (Patel and Patel, 2011). Quinazoline$4(3 \mathrm{H})$-one with substituted phenyl ring, bridged phenyl rings at position 3 was reported to be linked with anti-microbial activity (Abdel-Rahman et al., 1997; Hassan et al., 1991). It has also been found that substitution at 2 and 3 position of quinazoline derivative is having significant analgesic and anti-inflammatory activity (Hemlatha and Girija, 2011). In the present study, we attempt to design and synthesize isomeric new series of quinazoline-4-one/4thione derivatives by structural modifications retaining the essential structural features for the activity and evaluated for their anti-microbial, analgesic and anti-inflammatory activity in order to identify new candidates that may me potent and selective with lesser toxic effects.

\section{MATERIAL AND METHODS}

\section{Chemicals and Instrumentation}

The synthesis of the target compounds was accomplished as illustrated in the Figure 2. The compounds were synthesized according to the procedure given in the respective literature (Hemlatha and Girija, 2011; Adel et al., 2010; Ilangovan et al., 2010). All the reagents and solvents used in the study were of analytical grade purity and procured from Sigma Aldrich Pvt. Ltd. (India). The progress of the reaction was monitored by thin layered chromatography with hexane: ethyl acetate $(3: 2)$ as the mobile phase and performed on silica gel $60 \mathrm{~F}_{254}$ aluminium sheets (Merck Ltd., Germany) and the products were purified by recrystallization. Melting points were determined in open capillaries using Stuart SMP10 (Barloworld Scientific Ltd., UK), electrothermal melting point apparatus. IR spectra were recorded on Shimadzu 8400S FTIR (Schimadzu Corporation, Japan) Spectrophotometer using $\mathrm{KBr}$ pallets and were recorded in $\mathrm{cm}^{-1}$. ${ }^{1} \mathrm{H}$ NMR $(400.13 \mathrm{MHz})$ spectra were acquired on a Bruker Avance II-400 NMR Spectrophotometer using TMS as the internal standard and the chemical shifts were recorded in $\delta$. The mass spectrum was obtained on WATER ZQ-4000 mass spectrophotometer. Elemental analysis for $\mathrm{C}, \mathrm{H}$ and $\mathrm{N}$ were performed on PERKIN ELMER 2400 SERIES-II CHN Analyzer.

\section{EXPERIMENT}

\section{Method of synthesis}

\section{Synthesis of 7-chloro-2-phenyl-4H-benzo[d] [1,3] oxazin-4-one (I): (Intermediate)}

4-chloroanthranilic acid (0.01 mole) was dissolved in dry pyridine $(30 \mathrm{ml})$ by stirring slowly at room temperature. The solution was cooled to $0^{\circ} \mathrm{C}$ and a solution of benzoyl chloride $(0$. 02 mole) in dry pyridine $(30 \mathrm{ml})$ was added slowly with constant stirring. After this addition, the reaction mixture was further stirred for half an hour at room temperature and set aside for $1 \mathrm{hr}$. The pasty mass obtained was diluted with water $(50 \mathrm{ml})$ and treated with aqueous sodium bicarbonate solution. When the effervescence ceased, the precipitate obtained was filtered off and washed with water, dried and recrystallized from diluted ethanol (Ilangovan et al., 2010)

\section{General Procedure for the synthesis of compounds, IIa-IIJ}

7-chloro-2-phenyl-4H-benzo[d][1,3] oxazin-4-one $\quad(0.01$ mol) and substituted phenyl hydrazine derivatives /hydrazine hydrate/semicarbazide/thiosemicarbazide $(0.01 \mathrm{~mol})$ were refluxed for $3 \mathrm{hrs}$ in presence of glacial acetic acid. The reaction mixture was allowed to cool at room temperature. The crude product was recrystallized using absolute alcohol to afford the target compounds IIa-IIj (Hemlata et al., 2011). 


\section{General Procedure for the synthesis of compounds, IIIa-IIIj}

A mixture of 7-chloro-(3-amino/substituted phenyl amino)-2-phenyl quinazoline-4 (3H)-one/1-(7-chloro-4-oxo-2phenylquinazolin-3(4H)-yl-urea/thiourea $(10 \mathrm{mmol}, 2.70 \mathrm{~kg})$ and phosphorus penta sulphide (1 mmol, $2.43 \mathrm{~g}$ ) was heated under reflux in anhydrous xylene $(100 \mathrm{ml})$ for $12 \mathrm{hrs}$. The reaction mixture was filtered while hot, the solvent was evaporated and the residue was triturated with dimethyl sulphoxide $(10 \mathrm{ml})$ and filtered.

The clear filtrate was poured into ice water, dried and recrystallised from ethanol to afford the target compounds IIIa-IIIj (Adel et al., 2010).

Compound I (7-chloro-2-phenyl-4H-benzo[d] [1, 3] oxazin-4one)

White powder (Methanol); 4-chloroanthranilic acid (0.01 mol) was dissolved in dry pyridine $(30 \mathrm{ml})$ by stirring slowly at room temperature. The solution was cooled to $0^{\circ} \mathrm{C}$ and a solution of benzoyl chloride $(0.02 \mathrm{~mol})$ in dry pyridine $(30 \mathrm{ml})$ was added slowly with constant stirring. After this addition, the reaction mixture was further stirred for half an hour at room temperature and set aside for $1 \mathrm{hr}$. The pasty mass obtained was diluted with water $(50 \mathrm{ml})$ and treated with aqueous sodium bicarbonate solution. When the effervescence ceased, the precipitate obtained was filtered off and washed with water, dried and recrystallized from diluted ethanol as a white solid. (Yield: 61.2\%); m.p 156$158^{\circ} \mathrm{C}$; IR $\left(\mathrm{KBr} \mathrm{cm}{ }^{-1}\right)$ vmax: Ar-CH stretch $\left(3072 \mathrm{~cm}^{-1}\right), \mathrm{C}=\mathrm{O}$ $\left(1751 \mathrm{~cm}^{-1}\right), \mathrm{C}=\mathrm{N}\left(1592 \mathrm{~cm}^{-1}\right)$, cyclic C-O-C stretch $\left(1060 \mathrm{~cm}^{-1}\right)$, $\mathrm{C}-\mathrm{Cl}\left(680 \mathrm{~cm}^{-1}\right) ;{ }^{1} \mathrm{H}$ NMR (DMSO-d6, 400.13 MHz): $87.31-7.69$ (m, 5H, Ar-H), 7.52-8.20 (t, 5H, Ar-H); MS m/z: $262.12(\mathrm{M}+)$, $\mathrm{C}_{14} \mathrm{H}_{8} \mathrm{ClNO}_{2}$ (Calcd. 257.67); Anal calcd. (\%) C, 65.26; H, 3.13; N, 5.44; Found: C, 65.67; H, 3.52; N, 5.84.

\section{Compound $\mathrm{II}_{a}$ (7-chloro-2-phenyl-3-(phenylamino) quinazolin- 4(3H)-one)}

Reddish brown crystalline solid (methanol); 7-chloro-2phenyl-4H-benzo[d][1,3]oxazin-4-one $(0.01 \mathrm{~mol})$ and phenyl hydrazine derivatives hydrate $(0.01 \mathrm{~mol})$ were refluxed for $3 \mathrm{hrs}$ in presence of glacial acetic acid. The reaction mixture was allowed to cool at room temperature. The crude product was recrystallized using absolute alcohol as a reddish brown solid. (Yield:87.2\%); m.p. 135-145 ${ }^{0}$; IR $\left(\mathrm{KBr} \mathrm{cm}{ }^{-1}\right.$ ) vmax: $\mathrm{Ar}-\mathrm{CH}_{\text {stretch }}\left(3262 \mathrm{~cm}^{-1}\right), \mathrm{C}=\mathrm{O}\left(1664 \mathrm{~cm}^{-1}\right), \mathrm{C}=\mathrm{N}\left(1595 \mathrm{~cm}^{-}\right.$ $\left.{ }^{1}\right), \mathrm{N}_{-} \mathrm{NH}_{\text {stretch }}\left(3343 \mathrm{~cm}^{-1}\right), \mathrm{C}-\mathrm{Cl}\left(689 \mathrm{~cm}^{-1}\right),{ }^{1} \mathrm{H}$ NMR (DMSO-d 6 , 400.13MHz), $\delta$ 7.491-7.601 (m, 5H, Ar-H ), 7.781-8.115 (m, 5H, Ar-H), 7.907-8.723 (t, 3H, Ar-H), 3.466 (s, 1H, N-H); MS m/z: $365.11(\mathrm{M}+) ; \mathrm{C}_{20} \mathrm{H}_{14} \mathrm{ClN}_{3} \mathrm{O}$ (Calcd. 347.8); Anal calcd. (\%) C, 69.07; H, 4.06; N, 12.08; Found: C, 69.48; H, 4.48;N, 12.48.

\section{Compound $\mathrm{II}_{\mathrm{b}}$ (7-chloro-2-phenyl-3- (o-chloro-phenylamino) quinazolin-4 (3H)-one)}

White crystalline solid (methanol); 7-chloro-2-phenyl$4 \mathrm{H}$-benzo[d][1, 3]oxazin-4-one $(0.01 \mathrm{~mol})$ and 2-chloro phenyl hydrazine $(0.01 \mathrm{~mol})$ have been refluxed for $3 \mathrm{hrs}$ in presence of glacial acetic acid.

The reaction mixture was allowed to cool at room temperature. The crude product was recrystallized using absolute alcohol as white crystalline solid. (Yield: 57. 14\%); m.p 168$170^{0} \mathrm{C}$; IR $\left(\mathrm{KBr} \mathrm{cm}{ }^{-1}\right)$ vmax: $\operatorname{Ar}-\mathrm{CH}_{\text {stretch }}\left(3323 \mathrm{~cm}^{-1}\right), \mathrm{C}=\mathrm{O}(1665$ $\left.\mathrm{cm}^{-1}\right), \mathrm{C}=\mathrm{N}\left(1592 \mathrm{~cm}^{-1}\right), \mathrm{N}-\mathrm{NH}_{\text {stretch }}\left(3307 \mathrm{~cm}^{-1}\right), \mathrm{C}-\mathrm{Cl}\left(676 \mathrm{~cm}^{-1}\right)$, ${ }^{1} \mathrm{H}$ NMR (DMSO-d 6 , 400. 13MHz), $\delta$ 6.753-7.181 (m, 4H, Ar-H, ), 7.305-7.72 (t, 5H, Ar-H), 7.52-7.907 (t, 3H, Ar-H), $4.56(\mathrm{~s}, 1 \mathrm{H}$, $\mathrm{N}-\mathrm{H}$ ), MS, m/z:382.02 (M+); $\mathrm{C}_{20} \mathrm{H}_{13} \mathrm{C}_{12} \mathrm{~N}_{3} \mathrm{O}$ (Calcd. 382.24); Anal calcd. (\%) C, 62.84;H, 3.43;N, 10.99; Found: C, 63.24;H, 3.85;N, 11.35 .

\section{Compound $\mathrm{II}_{c}$ (7-chloro-2-phenyl-3- (o-methyl-phenylamino) quinazolin-4 (3H)-one)}

Light brown crystalline solid (methanol); 7-chloro-2phenyl-4H-benzo[d] [1,3]oxazin-4-one (0. $01 \mathrm{~mol})$ and 2-methyl phenyl hydrazine $(0.01 \mathrm{~mol})$ have been refluxed for $3 \mathrm{hrs}$ in presence of glacial acetic acid. The reaction mixture was allowed to cool at room temperature. The crude product was recrystallized using absolute alcohol as light brown crystalline solid. (Yield: 67\%); m.p 163-166 ${ }^{\circ}$; IR ( $\left.\mathrm{KBr} \mathrm{cm}{ }^{-1}\right)$ vmax: $\mathrm{Ar}-\mathrm{CH}_{\text {stretch }}(3242 \mathrm{~cm}$ $\left.{ }^{1}\right), \mathrm{C}=\mathrm{O}\left(1677 \mathrm{~cm}^{-1}\right), \mathrm{C}=\mathrm{N}\left(1654 \mathrm{~cm}^{-1}\right), \mathrm{N}-\mathrm{NH}_{\text {stretch }}\left(3309 \mathrm{~cm}^{-1}\right), \mathrm{C}-$ $\mathrm{CH}_{3}\left(2911 \mathrm{~cm}^{-1}\right),{ }^{1} \mathrm{H}$ NMR (DMSO-d6, 400.13MHz), $\delta 6.85-7.02$ (m, 4H, Ar-H), 7.282-7.621 (m, 5H, Ar-H), 7.653-7.91 (t, 3H, Ar$\mathrm{H}), 3.818(\mathrm{~s}, 1 \mathrm{H}, \mathrm{N}-\mathrm{H}), 2.51$ (s, 1H, Ar-CH3), MS, m/z: 361.23 $(\mathrm{M}+) ; \mathrm{C}_{20} \mathrm{H}_{13} \mathrm{Cl}_{2} \mathrm{~N}_{3} \mathrm{O}$ (Calcd. 361.82); Anal calcd. (\%) C, 69.71; H, 4.46; N, 11.61; Found: C, 70.12; H, 4.87; N, 12.04.

\section{Compound $\mathrm{II}_{d}$ (7-chloro-2-phenyl-3-(p-chloro-phenylamino) quinazolin-4 (3H)-one)}

Brown crystalline solid (methanol) 7-chloro-2-phenyl$4 \mathrm{H}$-benzo[d][1,3]oxazin-4-one $(0.01 \mathrm{~mol})$ and $p$-chloro phenyl hydrazine $(0.01 \mathrm{~mol})$ were refluxed for $3 \mathrm{hrs}$ in presence of glacial acetic acid. The reaction mixture was allowed to cool at room temperature. The crude product was recrystallized using absolute alcohol as brown crystalline solid. (Yield: 91.67\%); m. p 171$174^{0} \mathrm{C}$; IR $\left(\mathrm{cm}^{-1}\right) ; v_{\max } \operatorname{Ar}-\mathrm{CH}_{\text {stretch }}\left(3010 \mathrm{~cm}^{-1}\right), \mathrm{C}=\mathrm{O}\left(1665 \mathrm{~cm}^{-1}\right)$, $\mathrm{C}=\mathrm{N}\left(1594 \mathrm{~cm}^{-1}\right), \mathrm{N}-\mathrm{NH}_{\text {stretch }}\left(3240 \mathrm{~cm}^{-1}\right), \mathrm{C}-\mathrm{Cl}\left(698 \mathrm{~cm}^{-1}\right),{ }^{1} \mathrm{H}$ NMR (DMSO-d6, 400.13MHz), $\delta$ 6. 743-7.23 (m, 4H, Ar-H, ), 7.352-7.68 (t, 4H, Ar-H), 7.54-7.907 (t, 3H, Ar-H), 4.43 (s, 1H, N$\mathrm{H}), \mathrm{MS}, \mathrm{m} / \mathrm{z}: 382.07(\mathrm{M}+) ; \mathrm{C}_{20} \mathrm{H}_{13} \mathrm{Cl}_{2} \mathrm{~N}_{3} \mathrm{O}$ (Calcd. 382.24); Anal calcd. (\%) C, 62.84; H, 3.42; N, 10.99; Found: C, 63.14; H, 3.82; $\mathrm{N}, 11.12$.

\section{Compound $\mathrm{II}_{e}$ (7-chloro-2-phenyl-3- (p-bromo-phenylamino) quinazolin-4 (3H)-one)}

Brown crystalline solid (methanol); 7-chloro-2-phenyl$4 \mathrm{H}$-benzo[d][1,3]oxazin-4-one $(0.01 \mathrm{~mol})$ and $\mathrm{p}$-bromo phenyl hydrazine $(0.01 \mathrm{~mol})$ were refluxed for $3 \mathrm{hrs}$ in presence of glacial acetic acid. The reaction mixture was allowed to cool at room temperature. The crude product was recrystallized using absolute alcohol as brown crystalline solid. (Yield: 91.67\%); m.p 172 $175^{\circ} \mathrm{C} ; \lambda \max (\mathrm{nm}) 274$; IR $\left(\mathrm{KBr} \mathrm{cm}{ }^{-1}\right)$ vmax: $\mathrm{Ar}-\mathrm{CH}_{\text {stretch }}(3271$ 
$\left.\mathrm{cm}^{-1}\right), \mathrm{C}=\mathrm{O}\left(1691 \mathrm{~cm}^{-1}\right), \mathrm{C}=\mathrm{N}\left(1645 \mathrm{~cm}^{-1}\right), \mathrm{N}-\mathrm{NH}_{\text {stretch }}\left(3332 \mathrm{~cm}^{-1}\right)$, $\mathrm{C}-\mathrm{Br}\left(693 \mathrm{~cm}^{-1}\right),{ }^{1} \mathrm{H}$ NMR (DMSO-d6, 400.13MHz), $\delta 6.52-7.24$ (m, 5H, Ar-H), 7.43-7.62 (m, 4H, Ar-H), 7.42-7.85 (m, 3H, Ar-H), 4.42 (s, 1H, N-H), MS, m/z: 412.85 (M+); $\mathrm{C}_{20} \mathrm{H}_{13} \mathrm{BrClN}_{3} \mathrm{O}$ (Calcd. 426.69); Anal calcd. (\%) C, 56.3; H, 3.07; N, 9.85; Found: C, $56.72 ; \mathrm{H}, 3.48 ; \mathrm{N}, 10.25$.

\section{Compound $\mathrm{II}_{f}$ (7-chloro-2-phenyl-3- (p-nitro-phenylamino) quinazolin-4 (3H)-one)}

Reddish brown solid (methanol); 7-chloro-2-phenyl-4Hbenzo[d][1, 3]oxazin-4-one $(0.01 \mathrm{~mol})$ and p-nitro phenyl hydrazine $(0.01 \mathrm{~mol})$ were refluxed for $3 \mathrm{hrs}$ in presence of glacial acetic acid. The reaction mixture was allowed to cool at room temperature. The crude product was recrystallized using absolute alcohol as brown crystalline solid. Yield: $80 \%$ ); m.p $171-173^{\circ} \mathrm{C}$; IR $\left(\mathrm{KBr} \mathrm{cm}{ }^{-1}\right)$ vmax: Ar- $\mathrm{CH}_{\text {stretch }}\left(3250 \mathrm{~cm}^{-1}\right), \mathrm{C}=\mathrm{O}\left(1650 \mathrm{~cm}^{-1}\right)$, $\mathrm{C}=\mathrm{N}\left(1592 \mathrm{~cm}^{-1}\right), \mathrm{N}-\mathrm{NH}_{\text {stretch }}\left(3341 \mathrm{~cm}^{-1}\right), \mathrm{C}-\mathrm{Cl}\left(682 \mathrm{~cm}^{-1}\right),{ }^{1} \mathrm{H}$ NMR (DMSO-d 6 , 400.13MHz), $\delta 6.94-7.31$ (t, 5H, Ar-H), 6.968.12 (m, 5H, Ar-H), 7.5-7.81 (t, 3H, Ar-H), 3.90 (s, 1H, N-H), MS, m/z: $378.15(\mathrm{M}+) ; \mathrm{C}_{20} \mathrm{H}_{13} \mathrm{ClN}_{4} \mathrm{O}_{3}$ (Calcd. 392.8); Anal calcd. (\%) C, 61.16; H, 3.34; N, 14.26; Found: C, 62.12; H, 4.15; N, 14.67.

\section{Compound $\mathrm{II}_{g}$ (7-chloro-2-phenyl-3- (p-methoxy-phenylamino) quinazolin-4 (3H)-one)}

Brownish yellow solid (methanol); 7-chloro-2-phenyl$4 \mathrm{H}$-benzo[d][1, 3]oxazin-4-one $(0.01 \mathrm{~mol})$ and p-methoxy phenyl hydrazine $(0.01 \mathrm{~mol})$ were refluxed for $3 \mathrm{hrs}$ in presence of glacial acetic acid. The reaction mixture was allowed to cool at room temperature.

The crude product was recrystallized using absolute alcohol as brown yellow solid. Yield: $92 \%)$; m. p $170-173^{\circ} \mathrm{C}$; IR $\left(\mathrm{KBr} \mathrm{cm}{ }^{-1}\right)$ vmax: Ar- $\mathrm{CH}_{\text {stretch }}\left(3114 \mathrm{~cm}^{-1}\right), \mathrm{C}=\mathrm{O}\left(1752 \mathrm{~cm}^{-1}\right), \mathrm{C}=\mathrm{N}$ $\left(1664 \mathrm{~cm}^{-1}\right), \mathrm{N}-\mathrm{NH}_{\text {stretch }}\left(3310 \mathrm{~cm}^{-1}\right), \mathrm{OCH}_{3}-\mathrm{CH}_{\text {stretch }}\left(3008 \mathrm{~cm}^{-1}\right)$, Ar- $\mathrm{CH}_{\text {stretch }}\left(3271 \mathrm{~cm}^{-1}\right),{ }^{1} \mathrm{H}$ NMR (DMSO-d6, 400.13MHz), $\delta$ 6.50-6.70 (m, 4H, Ar-H), 7.28-7.62 (m, 5H, Ar-H), 7.46-7.921 (t, $3 \mathrm{H}, \mathrm{Ar}-\mathrm{H}), 4.10$ (s, 1H, N-H), 3.78 (s, 1H, Ar-OCH $)_{3}, \mathrm{MS}, \mathrm{m} / \mathrm{z}$ : $345.12(\mathrm{M}+) ; \mathrm{C}_{21} \mathrm{H}_{16} \mathrm{ClN}_{3} \mathrm{O}$ (Calcd. 361.82); Anal Calcd. (\%) $\mathrm{C}$, 66.76; H, 4.27; N, 11.12; Found: C, 67.16; H, 4.67; N, 11.54.

\section{Compound $\mathrm{II}_{h}$ (3-amino-7-chloro-2-phenyquinazolin-4- (3H)- one)}

Brown crystalline solid (methanol); 7-chloro-2-phenyl$4 \mathrm{H}$-benzo[d][1,3] oxazin-4-one $(0.01 \mathrm{~mol})$ and hydrazine hydrazine hydrate $(0.01 \mathrm{~mol})$ were refluxed for $3 \mathrm{hrs}$ in presence of glacial acetic acid. The reaction mixture was allowed to cool at room temperature.

The crude product was recrystallized using absolute alcohol as brown yellow solid. (Yield: 92\%); m. p $170-173^{\circ} \mathrm{C}$; $\lambda \max (\mathrm{nm}) 278$; IR $\left(\mathrm{KBr} \mathrm{cm}{ }^{-1}\right)$ vmax: $\operatorname{Ar}-\mathrm{CH}_{\text {stretch }}\left(3126 \mathrm{~cm}^{-1}\right)$, $\mathrm{C}=\mathrm{O}\left(1598 \mathrm{~cm}^{-1}\right), \mathrm{C}=\mathrm{N}\left(1552 \mathrm{~cm}^{-1}\right), \mathrm{N}-\mathrm{NH}_{\text {stretch }}\left(3283 \mathrm{~cm}^{-1}\right), \mathrm{C}-\mathrm{Cl}$ $\left(696 \mathrm{~cm}^{-1}\right),{ }^{1} \mathrm{H}$ NMR (DMSO-d6, 400.13MHz), $\delta$ 7.21-7.56 (m, 5H, Ar-H), 7.42-7.87 (m, 3H, Ar-H), 2.40 (s, 1H, N-H), MS, m/z: $272.12(\mathrm{M}+) ; \mathrm{C}_{14} \mathrm{H}_{10} \mathrm{ClN}_{3} \mathrm{O}$ (Calcd. 271.7); Anal calcd. (\%) $\mathrm{C}$, 61.89; H, 3.71; N, 15.47; Found: C, 62.29; H, 4.12; N, 15.87.

\section{Compound $\mathrm{II}_{i}$ (1- (7-chloro-4-oxo-2-phenylquinazoline-3 (4H- yl)) urea)}

White crystalline solid (methanol); 7-chloro-2-phenyl$4 \mathrm{H}$-benzo[d][1, 3] oxazin-4-one $(0.01 \mathrm{~mol})$ and semicarbazide $(0$. $01 \mathrm{~mol}$ ) were refluxed for $3 \mathrm{hrs}$ in presence of glacial acetic acid. The reaction mixture was allowed to cool at room temperature. The crude product was recrystallized using absolute alcohol as white crystalline solid. (Yield: 60\%); m.p 165-167 ${ }^{\circ} \mathrm{C}$; IR $(\mathrm{KBr}$ $\left.\mathrm{cm}^{-1}\right)$ vmax: Ar- $\mathrm{CH}_{\text {stretch }}\left(3199 \mathrm{~cm}^{-1}\right), \mathrm{C}=\mathrm{O}\left(1671 \mathrm{~cm}^{-1}\right), \mathrm{C}=\mathrm{N}(1593$ $\left.\mathrm{cm}^{-1}\right), \mathrm{N}-\mathrm{NH}_{\text {stretch }}\left(3027 \mathrm{~cm}^{-1}\right), \mathrm{C}-\mathrm{Cl}\left(692 \mathrm{~cm}^{-1}\right),{ }^{1} \mathrm{H}$ NMR (DMSO$\mathrm{d} 6,400.13 \mathrm{MHz}), \delta 7.25-7.58(\mathrm{~m}, 5 \mathrm{H}, \mathrm{Ar}-\mathrm{H}), 7.37-7.86(\mathrm{~m}, 3 \mathrm{H}$, Ar-H), 5.80 (s, 1H, N-H), MS, m/z: $312.45\left(\mathrm{M}^{+}\right) ; \mathrm{C}_{15} \mathrm{H}_{11} \mathrm{ClN}_{4} \mathrm{O}_{2}$ (Calcd. 314.72); Anal calcd. (\%) C, 57.24; H, 3.52; N, 17.8; Found: C, 57.46; H, 3.94 ; N, 18.21.

\section{Compound $\mathrm{II}_{j}$ (1- (7-chloro-4-oxo-2-phenylquinazoline-3 (4H- yl))thiourea)}

White amorphous solid (methanol); 7-chloro-2-phenyl$4 \mathrm{H}$-benzo[d][1,3] oxazin-4-one $(0.01 \mathrm{~mol})$ and thiosemicarbazide $(0.01 \mathrm{~mol})$ were refluxed for $3 \mathrm{hrs}$ in presence of glacial acetic acid. The reaction mixture was allowed to cool at room temperature.

The crude product was recrystallized using absolute alcohol as white crystalline solid. (Yield: $70 \%$ ); m. p $162-165^{\circ} \mathrm{C}$; IR $\left(\mathrm{KBr} \mathrm{cm}{ }^{-1}\right)$ vmax: Ar- $\mathrm{CH}_{\text {stretch }}\left(3126 \mathrm{~cm}^{-1}\right), \mathrm{C}=\mathrm{O}\left(1644 \mathrm{~cm}^{-1}\right)$, $\mathrm{C}=\mathrm{N}\left(1552 \mathrm{~cm}^{-1}\right), \mathrm{N}-\mathrm{NH}_{\text {stretch }}\left(3432 \mathrm{~cm}^{-1}\right), \mathrm{C}-\mathrm{Cl}\left(755 \mathrm{~cm}^{-1}\right){ }^{1} \mathrm{H}$ NMR (DMSO-d6, 400.13MHz), $\delta$ 7.24-7.50 (m, 5H, Ar-H), 7.327.78 (m, 3H, Ar-H), 2.30 (s, 1H, N-H), MS, m/z: 332. 15 (M+); $\mathrm{C}_{15} \mathrm{H}_{11} \mathrm{ClN}_{4} \mathrm{OS}$ (Calcd. 330.79); Anal calcd. (\%) C, 54.46; H, 3.35; N, 16.94; Found: C, 54.86; H, 3.35; N, 16.94.

\section{Compound III $_{a}$ (7-chloro-2-phenyl-3- (phenylamino)-quinazolin- 4 (3H)-thione)}

Light brown crystalline solid (methanol); A mixture of 7chloro-2-phenyl-3- (phenylamino) quinazolin-4 (3H)-one (10 mmol, $2.70 \mathrm{~g}$ ) and phosphorus penta sulphide (1 mmol, $2.43 \mathrm{~g})$ was heated under reflux in anhydrous xylene $(100 \mathrm{ml})$ for $12 \mathrm{hrs}$. The reaction mixture was filtered while hot, the solvent was evaporated and the residue was triturated with dimethyl sulphoxide $(10 \mathrm{ml})$ and filtered. The clear filtrate was poured into ice water, dried and recrystallised from ethanol to form light brown crystalline solid. (Yield: $68 \%$ ); m.p. $165-168^{\circ} \mathrm{C}$; IR $\left(\mathrm{KBr} \mathrm{cm}^{-1}\right)$ vmax: Ar- $\mathrm{CH}_{\text {stretch }}\left(3025 \mathrm{~cm}^{-1}\right), \mathrm{C}-\mathrm{N}\left(1150 \mathrm{~cm}^{-1}\right), \mathrm{C}=\mathrm{N}\left(1666 \mathrm{~cm}^{-1}\right)$, $\mathrm{C}=\mathrm{S}\left(1262 \mathrm{~cm}^{-1}\right), \mathrm{C}-\mathrm{Cl}\left(735 \mathrm{~cm}^{-1}\right), \mathrm{N}-\mathrm{NH}$ (bend) $\left(3057 \mathrm{~cm}^{-1}\right),{ }^{1} \mathrm{H}$ NMR (DMSO-d6, 400.13MHz), $\delta$ 6.50-7.40 (m, 5H, Ar-H), 6.427.71 (m, 5H, Ar-H), 7.15-7.50 (m, 3H, Ar-H), 3.8 (s, 1H, N-H), MS, m/z: $362.15\left(\mathrm{M}^{+}\right) ; \mathrm{C}_{20} \mathrm{H}_{14} \mathrm{ClN}_{3} \mathrm{~S}$ (Calcd. 363.86); Anal calcd. (\%) C, 66.02; H, 3.88; N, 11.55; Found: C, 66.42; H, 4.28; N, 11.96 .

\section{Compound $\mathrm{III}_{\mathrm{b}}$ (7-chloro-2-phenyl-3- (o-chlorophenylamino)- quinazolin-4 (3H)-thione)}

Brown crystalline solid (methanol); A mixture of 7chloro-2-phenyl-3- (o-chloro-phenylamino) quinazolin-4 $(3 \mathrm{H})$-one 
(10 mmol, $2.70 \mathrm{~g}$ ) and phosphorus penta sulphide (1 mmol, $2.43 \mathrm{~g}$ ) was heated under reflux in anhydrous xylene $(100 \mathrm{ml})$ for $12 \mathrm{hrs}$. The reaction mixture was filtered while hot, the solvent was evaporated and the residue was triturated with dimethyl sulphoxide $(10 \mathrm{ml})$ and filtered. The clear filtrate was poured into ice water, dried and recrystallised from ethanol to form brown crystalline solid. (Yield: $67 \%$ ); m.p. $140-142^{\circ} \mathrm{C}$; IR $\left(\mathrm{KBr} \mathrm{cm}^{-1}\right)$ vmax: Ar$\mathrm{CH}_{\text {stretch }}\left(3010 \mathrm{~cm}^{-1}\right), \mathrm{C}=\mathrm{S}\left(1665 \mathrm{~cm}^{-1}\right), \mathrm{C}=\mathrm{N}\left(1594 \mathrm{~cm}^{-1}\right), \mathrm{N}-$ $\mathrm{NH}_{\text {stretch }}\left(3240 \mathrm{~cm}^{-1}\right), \mathrm{C}-\mathrm{Cl}\left(698 \mathrm{~cm}^{-1}\right),{ }^{1} \mathrm{H}$ NMR (DMSO-d6, 400.13MHz), $\delta 6.62-7.35$ (m, 5H, Ar-H), 6.45-7.68 (m, 5H, Ar-H), 7.2-7.48 (m, 3H, Ar-H), 4.10 (s, 1H, N-H), MS, m/z: 396.14 (M+); $\mathrm{C}_{14} \mathrm{H}_{10} \mathrm{ClN}_{3} \mathrm{~S}$ (Calcd. 398.31); Anal calcd. (\%) C, 60.31; H, 3.29; N, 10.55; Found: C, 60.71; H, 3.59; N, 10.97.

\section{Compound III (7-chloro-2-phenyl-3-(o-methylphenylamino)- quinazolin-4 (3H)-thione)}

Brownish yellow crystalline solid (methanol); A mixture of 7-chloro-2-phenyl-3- (o-methyl-phenylamino) quinazolin-4 (3H)-one (10 mmol, 2.70g) and phosphorus penta sulphide (1 mmol, $2.43 \mathrm{~g})$ was heated under reflux in anhydrous xylene (100 $\mathrm{ml}$ ) for $12 \mathrm{hr}$.

The reaction mixture was filtered while hot, the solvent was evaporated and the residue was triturated with dimethyl sulphoxide $(10 \mathrm{ml})$ and filtered. The clear filtrate was poured into ice water, dried and recrystallized from ethanol to form brownish yellow crystalline solid. (Yield: $71 \%)$; m. p $172-175^{\circ} \mathrm{C}$; IR $(\mathrm{KBr}$ $\left.\mathrm{cm}^{-1}\right)$ vmax: $\mathrm{Ar}-\mathrm{CH}_{\text {stretch }}\left(3114 \mathrm{~cm}^{-1}\right), \mathrm{C}=\mathrm{S}\left(1264 \mathrm{~cm}^{-1}\right), \mathrm{C}=\mathrm{N}(1664$ $\left.\mathrm{cm}^{-1}\right), \mathrm{C}-\mathrm{N}\left(1099 \mathrm{~cm}^{-1}\right) \mathrm{N}-\mathrm{NH}_{\text {stretch }}\left(3310 \mathrm{~cm}^{-1}\right), \mathrm{CH}_{3}-\mathrm{CH}_{\text {stretch }}(3008$ $\mathrm{cm}^{-1}$ ), ${ }^{1} \mathrm{H}$ NMR (DMSO-d6, 400.13 MHz), $\delta$ 6.50-7.57 (m, 5H, ArH), 6.67-7.58 (m, 5H, Ar-H), 7.31-7.35 (t, 3H, Ar-H), 3.90 (s, 1H, $\mathrm{N}-\mathrm{H}), 2.34$ (s, 1H, Ar-CH3), MS, m/z: $378.19(\mathrm{M}+) \mathrm{C}_{20} \mathrm{H}_{13} \mathrm{Cl}_{2} \mathrm{~N}_{3} \mathrm{~S}$ (Calcd. 377.89); Anal calcd. (\%) C, 66.75; H, 4.27; N, 10.55; Found: C, 60.71; H, 3.47; N, 11.44 .

\section{Compound $\mathrm{III}_{d}$ (7-chloro-2-phenyl-3-(p-chlorophenylamino)- quinazolin-4 (3H)-thione)}

Dark brown crystalline solid (methanol); A mixture of 7chloro-2-phenyl-3- (p-chloro-phenylamino) quinazolin-4 $(3 \mathrm{H})$-one (10 mmol, $2.70 \mathrm{~g}$ ) and phosphorus penta sulphide (1 mmol, $2.43 \mathrm{~g}$ ) was heated under reflux in anhydrous xylene $(100 \mathrm{ml})$ for $12 \mathrm{hrs}$. The reaction mixture was filtered while hot, the solvent was evaporated and the residue was triturated with dimethyl sulphoxide $(10 \mathrm{ml})$ and filtered. The clear filtrate was poured into ice water, dried and recrystallized from ethanol to form dark brown crystalline solid. (Yield: $72 \%)$; m. p. $162-163^{\circ} \mathrm{C}$; IR $\left(\mathrm{KBr} \mathrm{cm}^{-1}\right)$ vmax: Ar- $\mathrm{CH}_{\text {stretch }}\left(3029 \mathrm{~cm}^{-1}\right), \mathrm{C}-\mathrm{N}\left(1171 \mathrm{~cm}^{-1}\right), \mathrm{C}=\mathrm{N}\left(1676 \mathrm{~cm}^{-1}\right)$, $\mathrm{C}=\mathrm{S}\left(1263 \mathrm{~cm}^{-1}\right), \mathrm{C}-\mathrm{Cl}\left(761 \mathrm{~cm}^{-1}\right), \mathrm{N}-\mathrm{NH}$ (bend) $\left(3196 \mathrm{~cm}^{-1}\right),{ }^{1} \mathrm{H}$ NMR (DMSO-d6, 400.13MHz), $\delta$ 6.58-7.24 (m, 5H, Ar-H), 6.707.34 (m, 5H, Ar-H), 7.25-7.42 (m, 3H, Ar-H), 4.20 (s, 1H, N-H), MS, m/z: $398.05(\mathrm{M}+) ; \mathrm{C}_{20} \mathrm{H}_{13} \mathrm{Cl}_{2} \mathrm{~N}_{3} \mathrm{~S}$ (Calcd. 398.31); Anal calcd. (\%) C, 60.31; H, 3.29; N, 11.12; Found: C, 67.12; H, 4.67; N, 11.02 .
Compound $\mathrm{III}_{e}$ (3- (4-bromophenylamino)-7-chloro-2-phenylquinazolin-4 (3H)-thione)

Brownish yellow crystalline solid (methanol); A mixture of 7-chloro-2-phenyl-3- (p-bromo-phenylamino) quinazolin-4 (3H)-one (10 mmol, $2.70 \mathrm{~g})$ and phosphorus penta sulphide (1 mmol, $2.43 \mathrm{~g}$ ) was heated under reflux in anhydrous xylene (100 $\mathrm{ml}$ ) for $12 \mathrm{hrs}$. The reaction mixture was filtered while hot, the solvent was evaporated and the residue was triturated with dimethyl sulphoxide $(10 \mathrm{ml})$ and filtered. The clear filtrate was poured into ice water, dried and recrystallized from ethanol to form brownish yellow crystalline solid. (Yield: 69\%); m.p. 170$173^{\circ} \mathrm{C}$; IR $\left(\mathrm{KBr} \mathrm{cm}{ }^{-1}\right)$ vmax: Ar- $\mathrm{CH}_{\text {stretch }}\left(2918 \mathrm{~cm}^{-1}\right), \mathrm{C}=\mathrm{S}(1233$ $\left.\mathrm{cm}^{-1}\right), \mathrm{C}=\mathrm{N}\left(1665 \mathrm{~cm}^{-1}\right), \mathrm{N}-\mathrm{NH}_{\text {stretch }}\left(3060 \mathrm{~cm}^{-1}\right), \mathrm{C}-\mathrm{Br}\left(683 \mathrm{~cm}^{-1}\right)$, ${ }^{1} \mathrm{H}$ NMR (DMSO-d6, 400.13MHz), $\delta$ 6.53-7.58 (m, 5H, Ar-H, ), 7.43-7.62 (m, 4H, Ar-H), 7.24-7.41 (m, 3H, Ar-H), 4.20 (s, 1H, N$\mathrm{H}), \mathrm{MS}, \mathrm{m} / \mathrm{z}: 445.82(\mathrm{M}+) ; \mathrm{C}_{20} \mathrm{H}_{13} \mathrm{BrClN}_{3} \mathrm{~S}$ (Calcd. 442.76); Anal calcd. (\%) C, 54.25; H, 2.96; N, 9.49; Found: C, 54.65; H, 3.37; N, 9.59 .

\section{Compound $\mathrm{III}_{f}$ (7-chloro-2-phenyl-3- (p-nitrophenylamino)- quinazolin-4 (3H)-thione)}

Reddish brown crystalline solid (methanol); A mixture of 7-chloro-2-phenyl-3- (p-nitro-phenylamino) quinazolin-4 $(3 \mathrm{H})$-one (10 mmol, $2.70 \mathrm{~g})$ and phosphorus penta sulphide (1 mmol, $2.43 \mathrm{~g})$ was heated under reflux in anhydrous xylene $(100 \mathrm{ml})$ for $12 \mathrm{hrs}$. The reaction mixture was filtered while hot, the solvent was evaporated and the residue was triturated with dimethyl sulphoxide $(10 \mathrm{ml})$ and filtered. The clear filtrate was poured into ice water, dried and recrystallized from ethanol to form reddish brown crystalline solid. (Yield: $70 \%)$; m. p $171-174{ }^{\circ} \mathrm{C}$; IR $\left(\mathrm{KBr} \mathrm{cm}^{-1}\right)$ vmax: $\mathrm{Ar}-\mathrm{CH}_{\text {stretch }}\left(3255 \mathrm{~cm}^{-1}\right), \mathrm{C}=\mathrm{O}\left(1648 \mathrm{~cm}^{-1}\right), \mathrm{C}=\mathrm{N}(1594 \mathrm{~cm}$ $\left.{ }^{1}\right), \mathrm{N}_{-} \mathrm{NH}_{\text {stretch }}\left(3342 \mathrm{~cm}^{-1}\right), \mathrm{C}-\mathrm{Cl}\left(692 \mathrm{~cm}^{-1}\right),{ }^{1} \mathrm{H}$ NMR (DMSO-d 6 , 400.13MHz), $\delta$ 7.12-7.56 (t, 5H, Ar-H, ), 6.95-8.20 (m, 5H, Ar-H), 7.31-7.42 (t, 3H, Ar-H), 3.89 (s, 1H, N-H), MS, m/z:407.16 (M+); $\mathrm{C}_{20} \mathrm{H}_{13} \mathrm{ClN}_{4} \mathrm{O}_{2} \mathrm{~S}$ (Calcd. 408.86); Anal calcd. (\%) C, 58.75; H, 3.20; N, 13.7; Found: C, 59.12; H, 3.62; N, 14.12.

\section{Compound $\mathrm{III}_{g}$ (7-chloro-2-phenyl-3- (p-methoxyphenylamino)- quinazolin-4 (3H)-thione)}

Light brown crystalline solid (methanol); A mixture of 7 chloro-2-phenyl-3- (p-methoxy-phenylamino) quinazolin-4 $(3 \mathrm{H})$ one (10 mmol, $2.70 \mathrm{~g})$ and phosphorus penta sulphide $(1 \mathrm{mmol}$, $2.43 \mathrm{~g}$ ) was refluxed in anhydrous xylene $(100 \mathrm{ml})$ for $12 \mathrm{hrs}$. The reaction mixture was filtered while hot, the solvent was evaporated and the residue was triturated with dimethyl sulphoxide $(10 \mathrm{ml})$ and filtered. The clear filtrate was poured into ice water, dried and recrystallized from ethanol to form Light brown crystalline solid. (Yield: $72 \%)$; m. p $172-174^{\circ} \mathrm{C}$; IR $\left(\mathrm{KBr} \mathrm{cm}{ }^{-1}\right)$ vmax: $\mathrm{Ar}-\mathrm{CH}_{\text {stretch }}$ $\left(3242 \mathrm{~cm}^{-1}\right), \mathrm{C}=\mathrm{S}\left(1261 \mathrm{~cm}^{-1}\right), \mathrm{C}=\mathrm{N}\left(1677 \mathrm{~cm}^{-1}\right), \mathrm{C}-\mathrm{N}\left(1193 \mathrm{~cm}^{-1}\right)$, $\mathrm{N}-\mathrm{NH}_{\text {stretch }}\left(3309 \mathrm{~cm}^{-1}\right), \mathrm{C}^{-\mathrm{CH}_{3}}\left(2911 \mathrm{~cm}^{-1}\right),{ }^{1} \mathrm{H}$ NMR (DMSO-d6, 400.13MHz), $\delta 6.54-7.52$ (m, 5H, Ar-H), 6.70-7.58 (m, 5H, Ar-H), 7.24-7.35 (m, 3H, Ar-H), 4.2 (s, 1H, N-H), MS, m/z: 338.15 (M+); 
$\mathrm{C}_{21} \mathrm{H}_{16} \mathrm{ClN}_{3} \mathrm{~S}$ (Calcd. 393.89); Anal calcd. (\%) C, 58.75; H, 3.20;

N, 13.7; Found: C, 59.12; H, 3.62; N, 14.12.

\section{Compound $\mathrm{III}_{h}$ (3-amino-7-chloro-2-phenyl-quinazoline-4- (3H)-thione)}

Reddish brown crystalline solid (methanol); A mixture of 3-amino-7-chloro-2-phenyquinazolin-4- (3H)-one (10 mmol, 2.70 g) and phosphorus penta sulphide (1 mmol, $2.43 \mathrm{~g}$ ) was reflux in anhydrous xylene $(100 \mathrm{ml})$ for $12 \mathrm{hrs}$. The reaction mixture was filtered while hot, the solvent was evaporated and the residue was triturated with dimethyl sulphoxide $(10 \mathrm{ml})$ and filtered. The clear filtrate was poured into ice water, dried and recrystallized from ethanol to form Light brown crystalline solid. (Yield: 65\%); m.p. 172-175 ${ }^{\circ}$; IR $\left(\mathrm{KBr} \mathrm{cm}{ }^{-1}\right)$ vmax: $\mathrm{Ar}-\mathrm{CH}_{\text {stretch }}\left(3262 \mathrm{~cm}^{-1}\right), \mathrm{C}-\mathrm{N}$ $\left(1152 \mathrm{~cm}^{-1}\right), \mathrm{C}=\mathrm{N}\left(1664 \mathrm{~cm}^{-1}\right), \mathrm{C}=\mathrm{S}\left(1274 \mathrm{~cm}^{-1}\right), \mathrm{C}-\mathrm{Cl}\left(747 \mathrm{~cm}^{-1}\right)$, $\mathrm{N}-\mathrm{NH}$ (bend) $\left(3343 \mathrm{~cm}^{-1}\right),{ }^{1} \mathrm{H}$ NMR (DMSO-d6, 400.13MHz), $\delta$ 7.24-7.62 (m, 5H, Ar-H), 7.23-7.41 (m, 3H, Ar-H), 2.30 (s, 1H, NH). MS, m/z: $288.12(\mathrm{M}+) ; \mathrm{C}_{21} \mathrm{H}_{16} \mathrm{ClN}_{3} \mathrm{OS}$ (Calcd. 287.77); Anal calcd. (\%) C, 58.43; H, 3.5; N, 14.6; Found: C, 58.89; H, 3.82; N, 15.05 .

\section{Compound III $_{i}$ (1- (7-chloro-2-phenyl-4-thioxoquinazoline-3- (4H)-urea)}

White crystalline solid (methanol); A mixture of 1- (7chloro-4-oxo-2-phenylquinazoline-3 (4H-yl)) urea (10 mmol, 2.70 g) and phosphorus penta sulphide (1 mmol, $2.43 \mathrm{~g})$ was heated under reflux in anhydrous xylene $(100 \mathrm{ml})$ for $12 \mathrm{hrs}$. The reaction mixture was filtered while hot, the solvent was evaporated and the residue was triturated with dimethyl sulphoxide $(10 \mathrm{ml})$ and filtered.

The clear filtrate was poured into ice water, dried and recrystallized from ethanol to form Light brown crystalline solid. (Yield: $64 \%)$; m.p $162-165^{\circ} \mathrm{C}$; IR $\left(\mathrm{KBr} \mathrm{cm}{ }^{-1}\right)$ vmax: $\mathrm{Ar}-\mathrm{CH}_{\text {stretch }}$ $\left(3126 \mathrm{~cm}^{-1}\right), \mathrm{C}=\mathrm{S}\left(1243 \mathrm{~cm}^{-1}\right), \mathrm{C}=\mathrm{N}\left(1598 \mathrm{~cm}^{-1}\right), \mathrm{C}-\mathrm{N}\left(1193 \mathrm{~cm}^{-1}\right)$ $\mathrm{N}-\mathrm{NH}_{\text {stretch }}\left(3283 \mathrm{~cm}^{-1}\right),{ }^{1} \mathrm{H}$ NMR (DMSO-d6, 400.13MHz), $\delta$ 7.24-7.63 (m, 5H, Ar-H), 7.24-7.42 (m, 3H, Ar-H), 6.20 (s, 1H, NH). MS, m/z: $332.12(\mathrm{M}+) ; \mathrm{C}_{15} \mathrm{H}_{11} \mathrm{ClN}_{4} \mathrm{OS}$ (Calcd. 330.79); Anal calcd. (\%)C, 54.46; H, 3.35; N, 16.94; Found: C, 54.86; H, 3.85; $\mathrm{N}, 17.32$.

\section{Compound $\mathrm{III}_{j}$ (1- (7-chloro-2-phenyl-4-thioxoquinazoline-3-} (4H)-thiourea)

White crystalline solid (methanol); A mixture of 1- (7chloro-4-oxo-2-phenylquinazoline-3 (4H-yl)) thiourea $(10 \mathrm{mmol}$, $2.70 \mathrm{~g})$ and phosphorus penta sulphide (1 mmol, $2.43 \mathrm{~g})$ was heated under reflux in anhydrous xylene $(100 \mathrm{ml})$ for $12 \mathrm{hrs}$. The reaction mixture was filtered while hot, the solvent was evaporated and the residue was triturated with dimethyl sulphoxide $(10 \mathrm{ml})$ and filtered. The clear filtrate was poured into ice water, dried and recrystallized from ethanol to form Light brown crystalline solid. (Yield: $75 \%)$; m.p. $163-165^{0} \mathrm{C}$; IR $\left(\mathrm{KBr} \mathrm{cm}{ }^{-1}\right)$ vmax: $\mathrm{Ar}-\mathrm{CH}_{\text {stretch }}$ (3199 $\left.\mathrm{cm}^{-1}\right), \mathrm{C}=\mathrm{S}\left(1266 \mathrm{~cm}^{-1}\right), \mathrm{C}=\mathrm{N}\left(1671 \mathrm{~cm}^{-1}\right), \mathrm{N}-\mathrm{NH}_{\text {stretch }}(3027$ $\left.\mathrm{cm}^{-1}\right), \mathrm{C}-\mathrm{Cl}\left(692 \mathrm{~cm}^{-1}\right),{ }^{1} \mathrm{H}$ NMR (DMSO-d6, 400.13MHz), $\delta 7.25-$ 7.54 (m, 5H, Ar-H), 7.31-7.42 (m, 3H, Ar-H), 2.30 (s, 1H, N-H).
MS, m/z: $345.12(\mathrm{M}+) ; \mathrm{C}_{15} \mathrm{H}_{11} \mathrm{ClN}_{4} \mathrm{~S}_{2}$ (Calcd. 346.86); Anal calcd. (\%) C, 51.94; H, 3.2; N, 16.15; Found: C, 52.34; H, 3.46; N, 16.55 .

\section{Pharmacological activity}

The present biological study was approved by the institute animal ethics committee (GIPS/IAEC/9). All the chemicals and solvents used for the pharmacological activity were purchased from Sigma-Aldrich. The newly synthesized compounds (IIa-IIIj) were tested for their anti-microbial, analgesic and anti-inflammatory activity.

\section{Anti-microbial activity (Disk diffusion method)}

The antimicrobial activities were performed by disk diffusion method (Table-1). The synthesized compounds were dissolved in dimethyl formamide (DMF) at $100 \mu \mathrm{g} / \mathrm{mL}$. Antibacterial activity against Staphylococcus aureus, Bacillus Subtilis (gram positive), Pseudomonas aeruginosa, Escheria coli, (gram negative). Anti-fungal activity was carried out against Aspergillous niger and Candida albicans under aseptic conditions. Ciprofloxacin and fluconazole $(50 \mu \mathrm{g} / \mathrm{ml})$ were used as standard drug for anti-bacterial and anti-fungal activity. The zone of inhibition was compared with the standard drug after $24 \mathrm{hrs}$ of incubation at $25^{\circ} \mathrm{C}$ for anti-bacterial activity and $48 \mathrm{hrs}$ at $32^{\circ} \mathrm{C}$ for anti-fungal activity (Maddeshiya and Agarwal, 2013).

\section{Analgesic activity: (Tail immersion method)}

The analgesic activity was determined by the tailimmersion method (Hemlatha and Girija, 2011). Swiss mice $(n=6)$ of either sex selected by random sampling technique. Diclofenac sodium at the dose of $20 \mathrm{mg} / \mathrm{kg}$ (I.P.) was used as a standard drug. The test compounds at a dose of $100 \mathrm{mg} / \mathrm{kg}$ in dimethylsulphoxide (DMSO) were administered i.p. The animals were held in position by a suitable restrainer with the tail extending out and the tail (up to $5 \mathrm{~cm}$ ) was taken dipped in a beaker of water maintained at $55 \pm 0.5^{\circ} \mathrm{C}$.

The time in sec taken to withdraw the tail clearly out of the water was taken as the reaction time. The first reading $0 \mathrm{~min}$ was taken immediately after the administration of the test compound and the subsequent reaction time was recorded at 15 , 30, 60 and $120 \mathrm{~min}$ after the administration of compounds. A cut off point $15 \mathrm{sec}$ was observed to prevent the tail damage. The percentage analgesic activity was calculated using the following formula and the results are presented in the table 2 .

$$
\text { PAA }=\frac{\mathrm{T}_{2}-\mathrm{T}_{1}}{\mathrm{~T}_{2}} \text {--- } 100
$$

Where $T_{1}$ is the reaction time (in sec) before treatment, $T_{2}$ is the reaction time (in sec) after treatment and PAA is the percentage analgesic activity. 
Table 1: Antimicrobial activity.

\begin{tabular}{|c|c|c|c|c|c|c|c|c|}
\hline \multicolumn{3}{|c|}{$\begin{array}{l}\text { Name of compound with their structural } \\
\text { modification }\end{array}$} & \multicolumn{3}{|c|}{$\begin{array}{c}\text { Antibacterial activity } \\
\text { (Zone of inhibition in } \mathrm{mm} \text { ) }\end{array}$} & \multicolumn{3}{|c|}{$\begin{array}{c}\text { Antifungal activity } \\
\text { (Zone of inhibition in } \mathrm{mm} \text { ) }\end{array}$} \\
\hline Compound Name & $\mathrm{R} / \mathrm{R}^{1}$ & $X$ & S. aureus & E. coli & B. subtilis & $\begin{array}{c}P . \\
\text { aeruginosa }\end{array}$ & A. niger & C. albicans \\
\hline $\begin{array}{c}\text { Standard } \\
\text { Ciprofloxacin } \\
(50 \mu \mathrm{g} / \mathrm{mL})\end{array}$ & --- & ---- & 17.45 & 13.5 & 15.64 & 18.26 & --- & --- \\
\hline $\begin{array}{c}\text { Standard } \\
\text { Fluconazole } \\
(50 \mu \mathrm{g} / \mathrm{mL}) \\
\end{array}$ & ---- & ---- & ---- & ---- & ---- & ---- & 21.76 & 16.42 \\
\hline $\begin{array}{c}\text { IIa } \\
(100 \mu \mathrm{g} / \mathrm{mL})\end{array}$ & & $\mathrm{O}$ & 12.24 & 8.28 & 10.24 & 12.24 & 14.43 & 10.27 \\
\hline $\begin{array}{c}\mathrm{IIb} \\
(100 \mu \mathrm{g} / \mathrm{mL})\end{array}$ & & $\mathrm{O}$ & 12.45 & 11.12 & 11.42 & 13.32 & 13.97 & 11.29 \\
\hline $\begin{array}{c}\text { IIc } \\
(100 \mu \mathrm{g} / \mathrm{mL})\end{array}$ & & $\mathrm{O}$ & 12.56 & 10.42 & 11.08 & 14.24 & 20.58 & 15.78 \\
\hline $\begin{array}{c}\text { IId } \\
(100 \mu \mathrm{g} / \mathrm{mL})\end{array}$ & & $\mathrm{O}$ & 13.25 & 8.76 & 10.51 & 13.52 & 14.72 & 10.56 \\
\hline $\begin{array}{c}\text { IIe } \\
(100 \mu \mathrm{g} / \mathrm{mL})\end{array}$ & & $\mathrm{O}$ & 14.12 & 9.1 & 9.24 & 12.88 & 13.86 & 11.57 \\
\hline $\begin{array}{c}\text { IIf } \\
(100 \mu \mathrm{g} / \mathrm{ml})\end{array}$ & & $\mathrm{O}$ & 11.28 & 9.23 & 10.12 & 12.95 & 14.75 & 12.98 \\
\hline $\begin{array}{c}\text { IIg } \\
(100 \mu \mathrm{g} / \mathrm{mL})\end{array}$ & & $\mathrm{O}$ & 16.54 & 8.75 & 14.76 & 14.57 & 20.42 & 15.24 \\
\hline IIh & $\mathrm{NH}_{2}$ & $\mathrm{O}$ & 13.42 & 9.32 & $14 . .82$ & 10.74 & 13.54 & 13.75 \\
\hline $\begin{array}{c}\text { IIi } \\
(100 \mu \mathrm{g} / \mathrm{mL})\end{array}$ & & $\mathrm{O}$ & 12.34 & 10.23 & 9.82 & 14.43 & 20.81 & 14.95 \\
\hline $\begin{array}{c}\mathbf{I I j} \\
(100 \mu \mathrm{g} / \mathrm{mL})\end{array}$ & & $\mathrm{O}$ & 11.67 & 10.12 & 11.32 & 14.64 & 20.56 & 15.82 \\
\hline $\begin{array}{c}\text { IIIa } \\
(100 \mu \mathrm{g} / \mathrm{mL})\end{array}$ & & $S$ & 10.24 & 9.85 & 10.91 & 11.12 & 13.74 & 12.87 \\
\hline $\begin{array}{c}\text { IIIb } \\
(100 \mu \mathrm{g} / \mathrm{mL})\end{array}$ & & S & 12.58 & 10.57 & 11.25 & 14.24 & 12.91 & 13.74 \\
\hline $\begin{array}{c}\text { IIIc } \\
(100 \mu \mathrm{g} / \mathrm{mL})\end{array}$ & & S & 13.14 & 10.32 & 10.87 & 14.35 & 14.72 & 12.82 \\
\hline $\begin{array}{c}\text { IIId } \\
(100 \mu \mathrm{g} / \mathrm{mL})\end{array}$ & & $S$ & 12.78 & 8.92 & 9.54 & 12.08 & 14.56 & 12.64 \\
\hline $\begin{array}{c}\text { IIIle } \\
(100 \mu \mathrm{g} / \mathrm{mL})\end{array}$ & & S & 12.61 & 9.24 & 10.34 & 11.75 & 14.91 & 10.51 \\
\hline
\end{tabular}




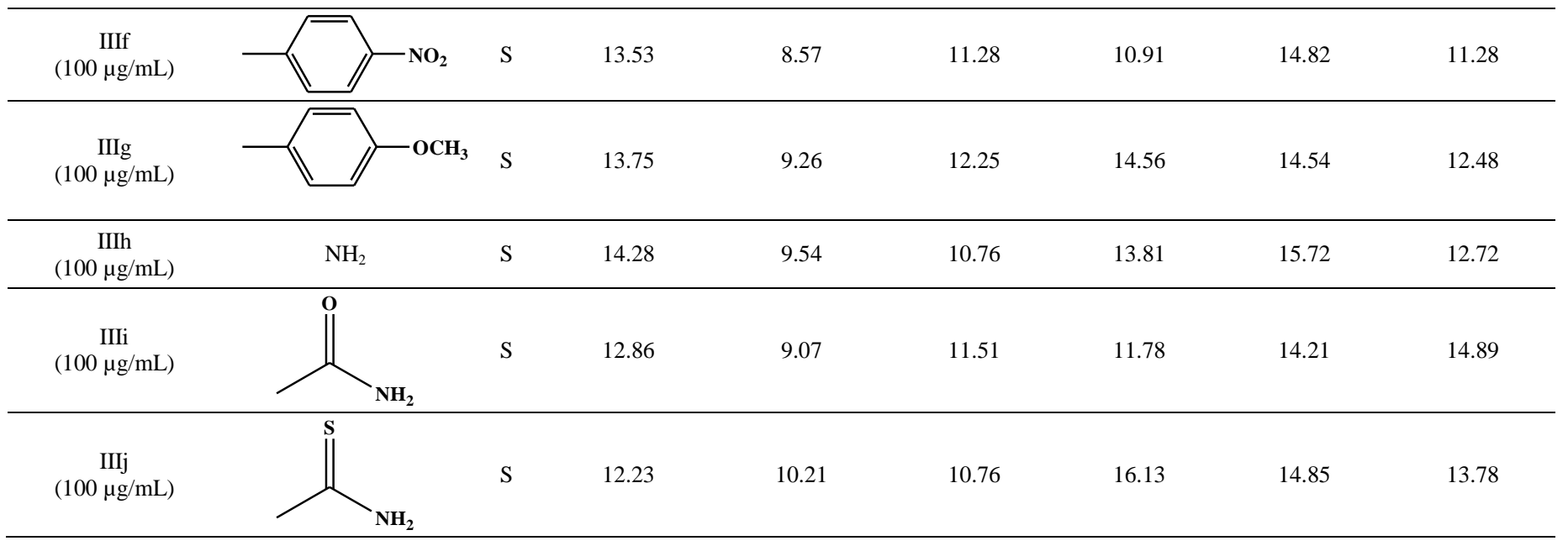

Table 2: Analgesic activity.

\begin{tabular}{|c|c|c|c|c|c|c|c|c|c|c|c|}
\hline \multirow[b]{3}{*}{ Compound } & \multirow[b]{3}{*}{$\mathbf{R} / \mathbf{R}^{1}$} & \multirow[b]{3}{*}{$\mathbf{X}$} & \multicolumn{9}{|c|}{ Basal Reaction Time in minutes } \\
\hline & & & \multirow{2}{*}{$\begin{array}{c}\text { 0 min } \\
\text { Mean } \pm \text { SEM }\end{array}$} & \multicolumn{2}{|c|}{$15 \mathrm{~min}$} & \multicolumn{2}{|c|}{$30 \mathrm{~min}$} & \multicolumn{2}{|c|}{$60 \mathrm{~min}$} & \multicolumn{2}{|c|}{$120 \mathrm{~min}$} \\
\hline & & & & Mean \pm SEM & $\%$ & Mean \pm SEM & $\%$ & Mean \pm SEM & $\%$ & $\begin{array}{c}\text { Mean } \pm \text { SE } \\
M\end{array}$ & $\%$ \\
\hline Control & ---- & --- & $1.25 \pm 0.01$ & $1.14 \pm 0.02$ & ---- & $1.15 \pm 0.01$ & ---- & $1.6 \pm 0.03$ & ---- & $1.56 \pm 0.03$ & ---- \\
\hline $\begin{array}{c}\text { Standard } \\
(20 \mathrm{mg} / \mathrm{kg})\end{array}$ & & $-\cdots$ & $1.78 \pm 0.07$ & $5.35 \pm 0.34$ & 66.72 & $8.5 \pm 0.15$ & 78.39 & $9.64 \pm 0.14$ & 81.53 & $4.34 \pm 0.3$ & 58.98 \\
\hline $\begin{array}{c}\text { IIa } \\
(100 \mathrm{mg} / \mathrm{kg})\end{array}$ & & $\mathrm{O}$ & $1.31 \pm 0.03$ & $2.85 \pm 0.02$ & 54.03 & $4.25 \pm 0.16$ & 69.17 & $4.68 \pm 0.12 * * *$ & 72.00 & $\begin{array}{c}2.64 \pm 0.12 * \\
* *\end{array}$ & 50.37 \\
\hline $\begin{array}{c}\mathrm{IIb} \\
(100 \mathrm{mg} / \mathrm{kg})\end{array}$ & & $\mathrm{O}$ & $1.34 \pm 0.04$ & $1.2 \pm 0.02$ & 57.05 & $4.85 \pm 0.15$ & 72.37 & $5.32 \pm 0.6^{* * *}$ & 74.81 & $\begin{array}{l}2.72 \pm 0.14 * \\
* *\end{array}$ & 50.73 \\
\hline $\begin{array}{c}\text { IIc } \\
(100 \mathrm{mg} / \mathrm{kg})\end{array}$ & & $\mathrm{O}$ & $1.56 \pm 0.02$ & $4.15 \pm 0.25$ & 62.4 & $6.42 \pm 0.12$ & 75.7 & $7.95 \pm 0.15 * * *$ & 80.37 & $\begin{array}{c}3.48 \pm 0.12 * \\
* *\end{array}$ & 55.17 \\
\hline $\begin{array}{c}\mathrm{IId} \\
(100 \mathrm{mg} / \mathrm{kg})\end{array}$ & & $\mathrm{O}$ & $1.32 \pm 0.03$ & $3.14 \pm 0.4$ & 57.96 & $4.72 \pm 0.17$ & 72.03 & $5.26 \pm 0.16^{* * *}$ & 74.90 & $\begin{array}{c}2.68 \pm 0.14 * \\
* *\end{array}$ & 50.74 \\
\hline $\begin{array}{c}\mathrm{Ile} \\
(100 \mathrm{mg} / \mathrm{kg})\end{array}$ & & $\mathrm{O}$ & $1.35 \pm 0.05$ & $3.15 \pm 0.18$ & 57.14 & $4.45 \pm 0.15$ & 69.66 & $5.36 \pm 0.16^{* * *}$ & 74.81 & $\begin{array}{c}2.72 \pm 0.05^{*} \\
* * \\
\end{array}$ & 50.36 \\
\hline $\begin{array}{c}\text { IIf } \\
(100 \mathrm{mg} / \mathrm{kg})\end{array}$ & & $\mathrm{O}$ & $1.28 \pm 0.04$ & $2.62 \pm 0.03$ & 51.14 & $4.21 \pm 0.15$ & 69.59 & $4.54 \pm 0.11 * * *$ & 71.8 & $\begin{array}{c}2.59 \pm 0.14 * \\
* *\end{array}$ & 50.57 \\
\hline $\begin{array}{c}\text { IIg } \\
(100 \mathrm{mg} / \mathrm{kg})\end{array}$ & & $\mathrm{O}$ & $1.52 \pm 0.04$ & $4.21 \pm 0.23$ & 63.89 & $6.44 \pm 0.14$ & 76.39 & $7.85 \pm 0.14 * * *$ & 80.89 & $\begin{array}{c}3.45 \pm 0.13 * \\
* *\end{array}$ & 55.94 \\
\hline $\begin{array}{c}\text { IIh } \\
(100 \mathrm{mg} / \mathrm{kg})\end{array}$ & $\mathrm{NH}_{2}$ & $\mathrm{O}$ & $1.30 \pm 0.02$ & $2.51 \pm 0.04$ & 48.20 & $4.26 \pm 0.12$ & 69.48 & $4.48 \pm 0.15 * * *$ & 70.98 & $\begin{array}{c}2.56 \pm 0.11 * \\
* *\end{array}$ & 49.21 \\
\hline $\begin{array}{c}\mathrm{IIi} \\
(100 \mathrm{mg} / \mathrm{kg})\end{array}$ & & $\mathrm{O}$ & $1.34 \pm 0.04$ & $2.62 \pm 0.14$ & 48.85 & $4.45 \pm 0.12$ & 69.88 & $4.52 \pm 0.15 * * *$ & 70.35 & $\begin{array}{c}2.62 \pm 0.05^{*} \\
* *\end{array}$ & $\begin{array}{c}48 . .8 \\
5\end{array}$ \\
\hline $\begin{array}{c}\mathrm{IIj} \\
(100 \mathrm{mg} / \mathrm{kg})\end{array}$ & & $\mathrm{O}$ & $1.32 \pm 0.03$ & $2.85 \pm 0.15$ & 53.68 & $4.25 \pm 0.19$ & 68.94 & $4.75 \pm 0.07 * * *$ & 72.21 & $\begin{array}{l}2.57 \pm 0.04 * \\
* *\end{array}$ & 48.63 \\
\hline $\begin{array}{c}\text { IIIa } \\
(100 \mathrm{mg} / \mathrm{kg})\end{array}$ & & S & $1.35 \pm 0.04$ & $2.52 \pm 0.02$ & 46.42 & $4.28 \pm 0.15$ & 68.45 & $4.46 \pm 0.12 * * *$ & 69.73 & $\begin{array}{c}2.48 \pm 0.13 * * \\
*\end{array}$ & 45.56 \\
\hline
\end{tabular}




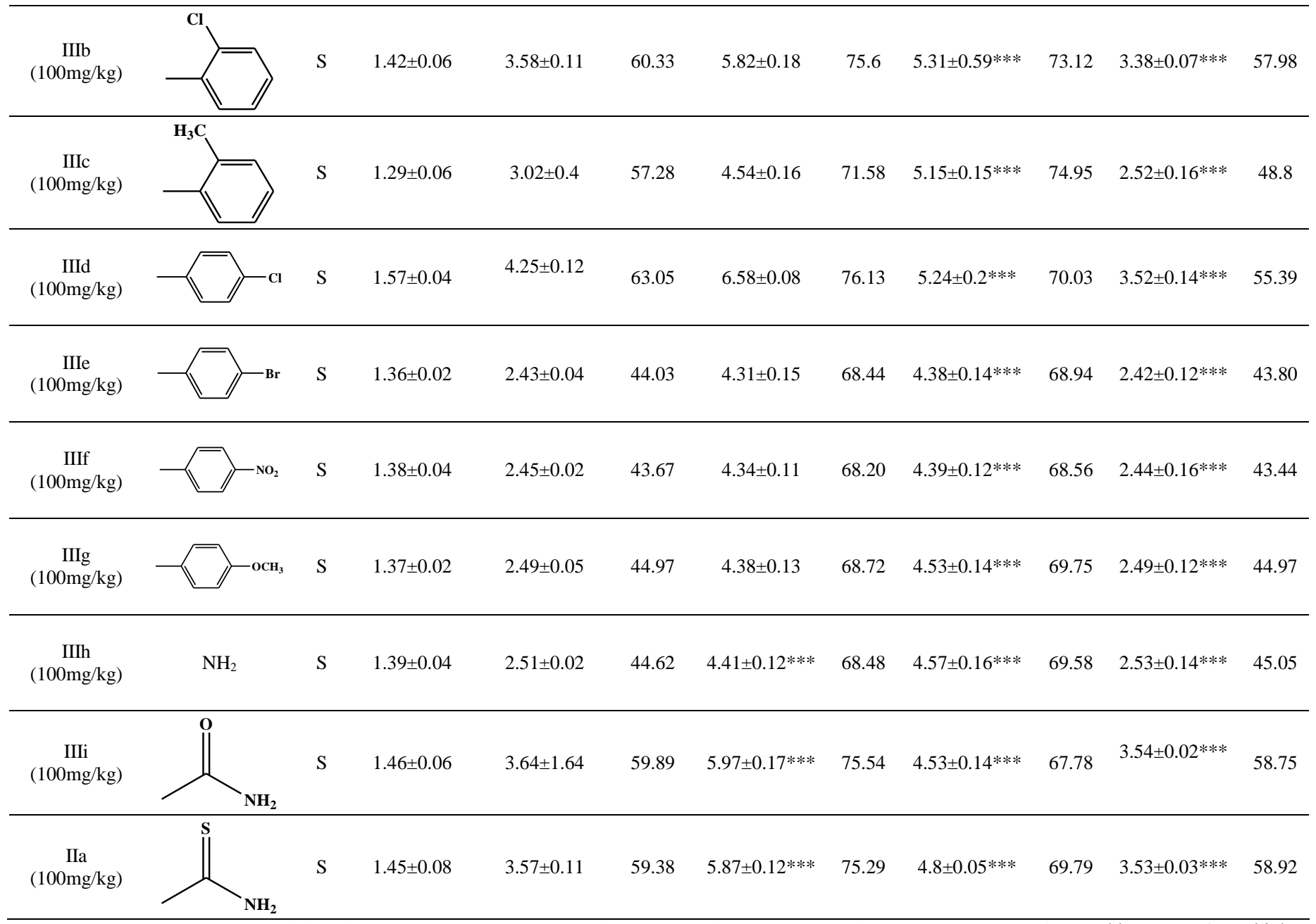

All the values are mean \pm SEM of six mice, where *** $p<0.05$ compared to control. All data are analyzed by one way ANOVA followed by Dunnett's Multiple Comparison Tests.

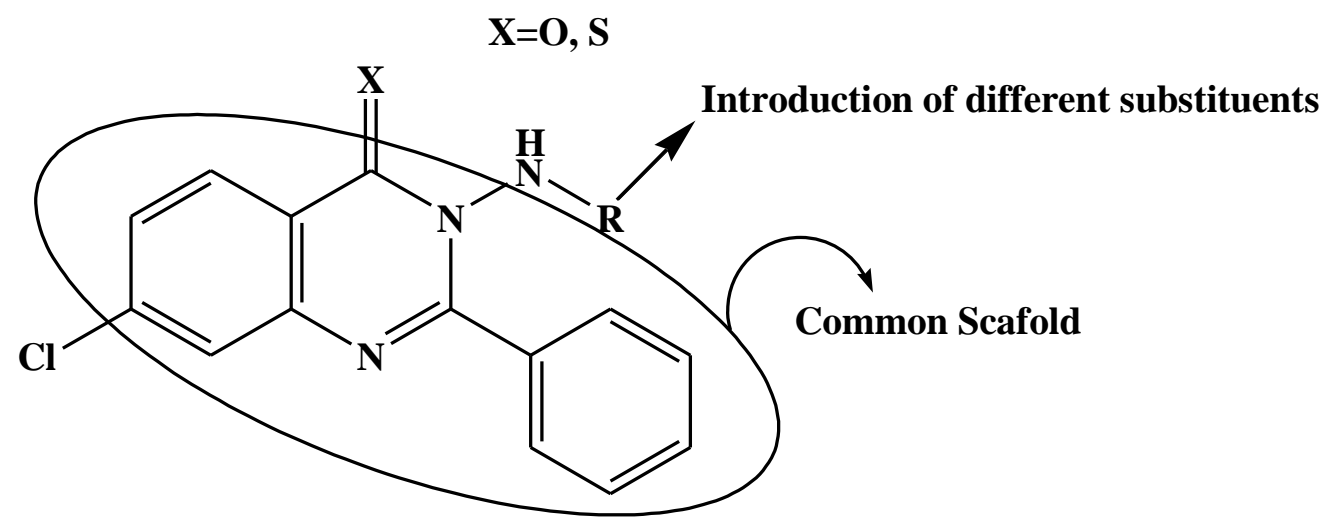

Fig. 1: Scaffold of the designed Quinazoline derivatives. 
Table 3: Anti inflammatory activity.

\begin{tabular}{|c|c|c|c|c|c|c|c|c|c|}
\hline \multirow{2}{*}{ Compound } & \multirow{2}{*}{$\mathbf{R} / \mathbf{R}^{1}$} & \multirow{2}{*}{$\mathbf{X}$} & \multicolumn{6}{|c|}{$\begin{array}{l}\text { Time interval in minutes } \\
(\text { Mean } \pm \text { S.E.M }) \% \text { inhibition }\end{array}$} & \multirow[b]{2}{*}{$\begin{array}{c}\% \\
\text { inhibition }\end{array}$} \\
\hline & & & 0 min & $15 \mathrm{~min}$ & $30 \mathrm{~min}$ & $60 \mathrm{~min}$ & $120 \mathrm{~min}$ & $180 \mathrm{~min}$ & \\
\hline Control & --- & $\begin{array}{ll}--- \\
\end{array}$ & $0.18 \pm 0.006$ & $0.2 \pm 0.0095$ & $0.24 \pm 0.007$ & $0.27 \pm 0.008$ & $0.31 \pm 0.009$ & $0.35 \pm 0.008$ & $\begin{array}{ll}--- \\
\end{array}$ \\
\hline $\begin{array}{c}\text { Standard } \\
\text { Diclofenac } \\
(20 \mathrm{mg} / \mathrm{kg})\end{array}$ & & ---- & $0.12 \pm 0.005$ & $0.14 \pm 0.007$ & $0.16 \pm 0.006$ & $0.19 \pm 0.008$ & $0.14 \pm 0.007$ & $0.10 \pm 0.005^{\mathrm{a}}$ & 71.42 \\
\hline $\begin{array}{c}\mathrm{IIa} \\
(100 \mathrm{mg} / \mathrm{kg})\end{array}$ & & $\mathrm{O}$ & $0.14 \pm 0.002$ & $0.16 \pm 0.0017^{\mathrm{a}}$ & $0.18 \pm 0.0056^{\mathrm{a}}$ & $0.2 \pm 0.0021^{\mathrm{a}}$ & $0.17 \pm 0.0047^{\mathrm{a}}$ & $0.15 \pm 0.004^{\mathrm{a}}$ & 57.14 \\
\hline $\begin{array}{c}\mathrm{IIb} \\
(100 \mathrm{mg} / \mathrm{kg})\end{array}$ & & $\mathrm{O}$ & $0.16 \pm 0.002$ & $0.17 \pm 0.004^{\mathrm{a}}$ & $0.2 \pm 0.003^{\mathrm{a}}$ & $0.18 \pm 0.006^{\mathrm{a}}$ & $0.15 \pm 0.005^{\mathrm{a}}$ & $0.14 \pm 0.004^{\mathrm{a}}$ & 60 \\
\hline
\end{tabular}

$\mathrm{IIc}$
$(100 \mathrm{mg} / \mathrm{kg})$

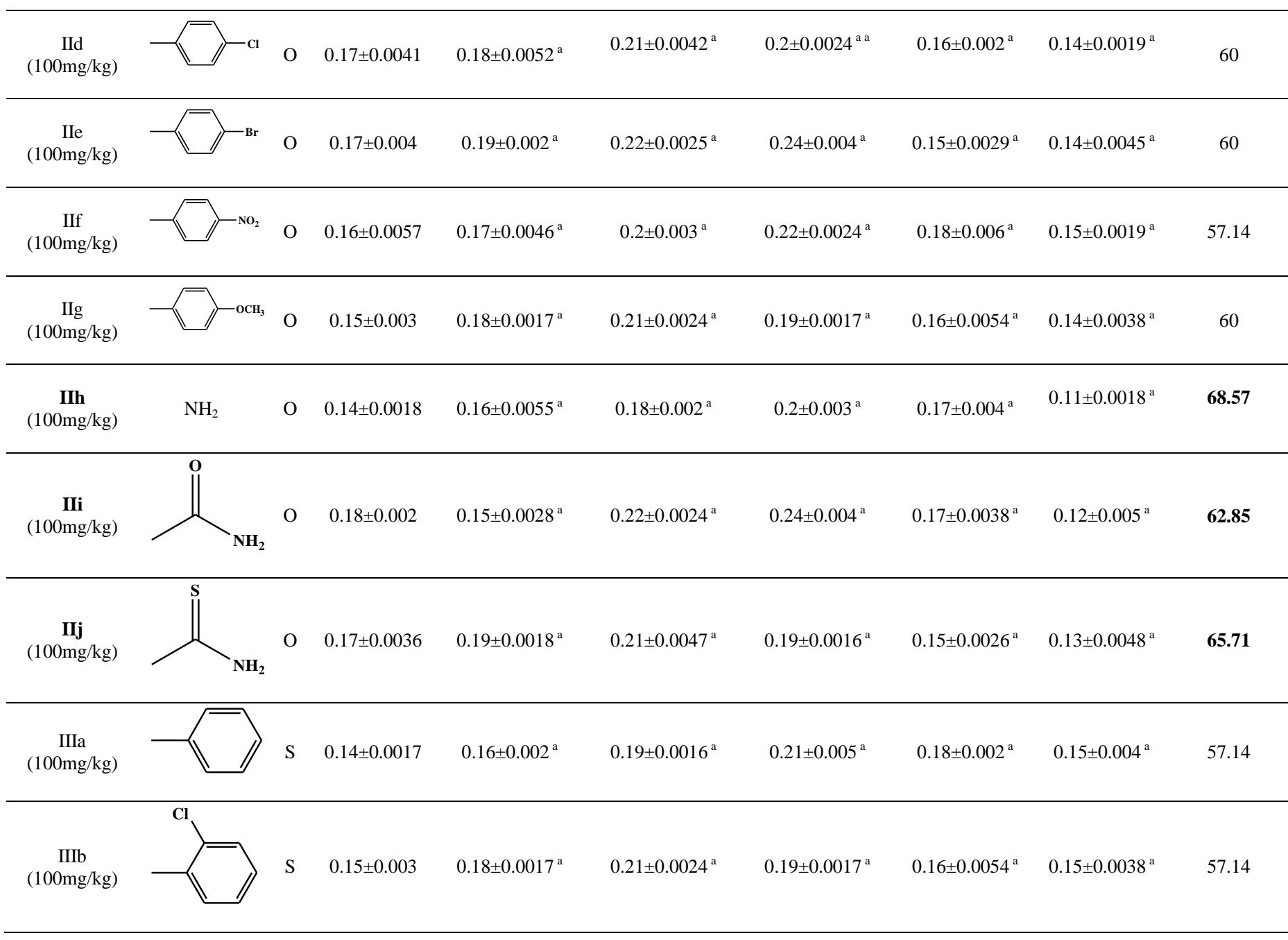




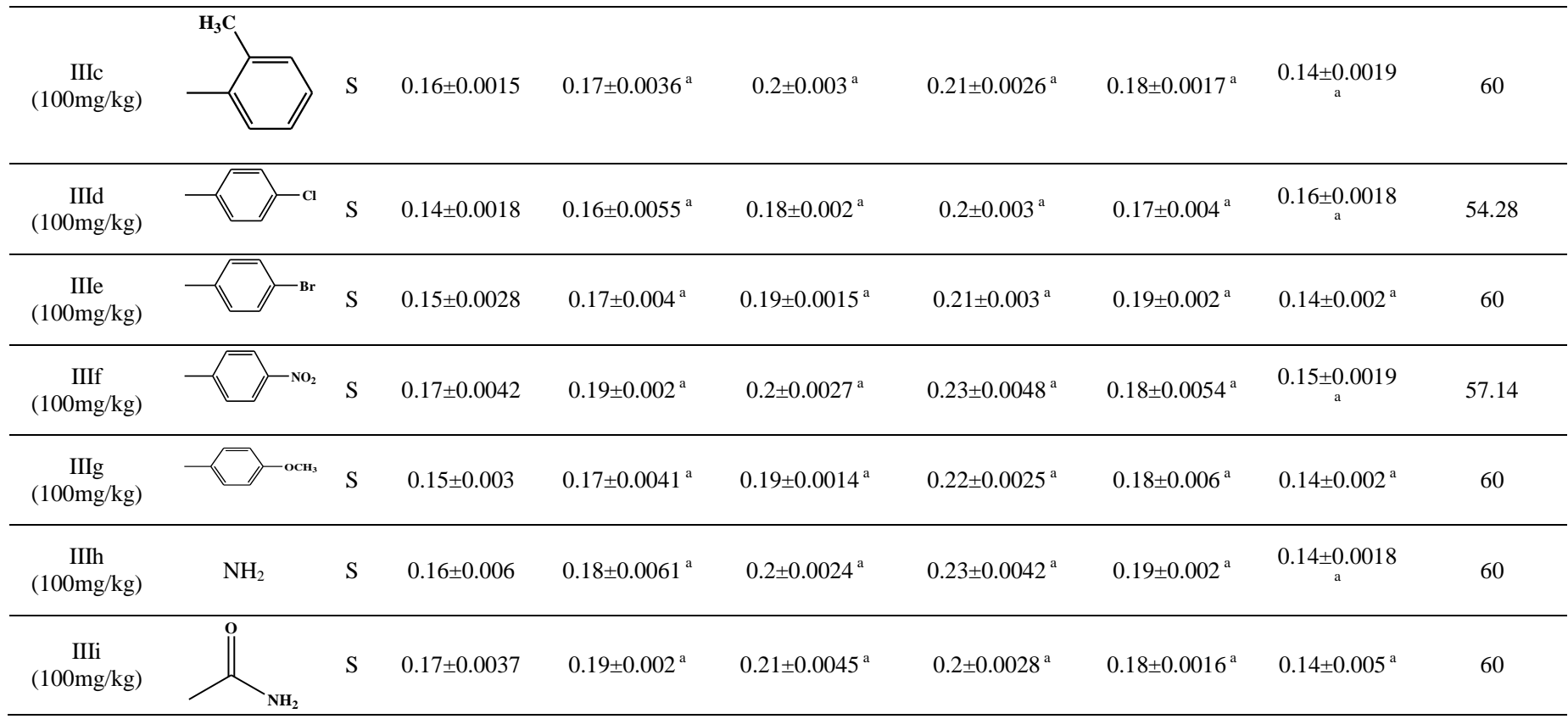

All the values are mean \pm SEM of six mice, where ${ }^{a} \mathrm{p}<0.05$ compared to control. All data are analyzed by one way ANOVA followed by Dunnett's Multiple Comparison Tests.

\section{Anti-inflammatory activity: (Carrageenan induced paw oedema method)}

Anti-inflammatory activity was performed by carrageenan induced paw oedema method in rats (Bekhit and Fahmy, 2003). Oedema was induced by sub-plantar injection of $0.1 \mathrm{ml}$ of freshly prepared $1 \%$ carrageenan into the right hind paw of the rats. The animals were divided into different groups of 6 each. Group-1 served as control and received carrageenan $(1 \% \mathrm{w} / \mathrm{v}$ in saline), group-2 served as standard and received diclofenac sodium (20 mg/kg i.p), and group 3 to 22 received the test compound $(100 \mathrm{mg} / \mathrm{kg})$. The volume of paw oedema was measured at $0,15,30,60,120$ and $180 \mathrm{~min}$ after injection of carrageenan using plethysmograph, the $\%$ of oedema inhibition was calculated for each animal group using the formula and the results were represented in the table 3.

$$
\% \text { Protection }=\text {----------------- } \text { x } 100
$$

\section{Acute toxicity study}

Approximate $50 \%$ lethal dose $\left(\mathrm{ALD}_{50}\right)$ of the most lead compounds was determined using standard procedures (Bekhit and Fahmy, 2003). Albino mice of either sex weighing 25-35 g were divided in 21 groups of 6 mice each. The synthesized compounds were dissolved in DMSO in 500, 750 and $1000 \mathrm{mg} / \mathrm{kg}$ doses were injected. The toxic symptoms and mortality rates in each group were recorded in $24 \mathrm{hrs}$ after drug administration.

\section{RESULT AND DISCUSSION}

\section{Spectral characterisation}

The proposed derivatives were synthesized as illustrated in the Figure 2. Physical properties and elemental analysis as well as all the spectral data are in accordance with the structure of the synthesized compounds.

The spectral data of I shows that Ar- $\mathrm{CH}_{\text {stretch }}\left(3072 \mathrm{~cm}^{-1}\right)$, $\mathrm{C}=\mathrm{O}\left(1751 \mathrm{~cm}^{-1}\right), \mathrm{C}=\mathrm{N}\left(1592 \mathrm{~cm}^{-1}\right)$, cyclic C-O-C $\mathrm{C}_{\text {stretch }}(1060 \mathrm{~cm}$ $\left.{ }^{1}\right), \mathrm{C}-\mathrm{Cl}\left(680 \mathrm{~cm}^{-1}\right)$. In addition, all the compounds displayed $\mathrm{C}_{4}-\mathrm{H}$ deformation. The mass spectra of the compounds were studied and the molecular ion peaks $(\mathrm{M}+)$, which were found consistent for all the compounds.

The elemental analyses were within $\pm \%$ of the theoretical values. ${ }^{1} \mathrm{H}$ NMR spectra of IIa - IIIj exhibited different spectral ranges in which each appears as multiplet and triplet due to the presence of non magnetically equivalent proton.

The aromatic protons show at the peak at $\delta 6.50-8.725$ ppm. The appearance of singlet protons around $\delta 3.46-4.50 \mathrm{ppm}$ for single protons in the ${ }^{1} \mathrm{H}$ NMR spectra might be assigned to $\mathrm{NH}$ - group.

The appearance of singlet proton $\delta$ 2.34-2.51 ppm for three protons in its ${ }^{1} \mathrm{H}$ NMR spectra which might be assigned to an aromatic methyl group confirms the formation of IIc/IIIc. The structures of the compounds are confirmed from the characteristics of the results obtained from analytical techniques. 
<smiles>Nc1cc(Cl)ccc1C(=O)O</smiles>

2-Amino-4-chloro benzoic acid

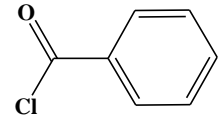

Benzoyl chloride
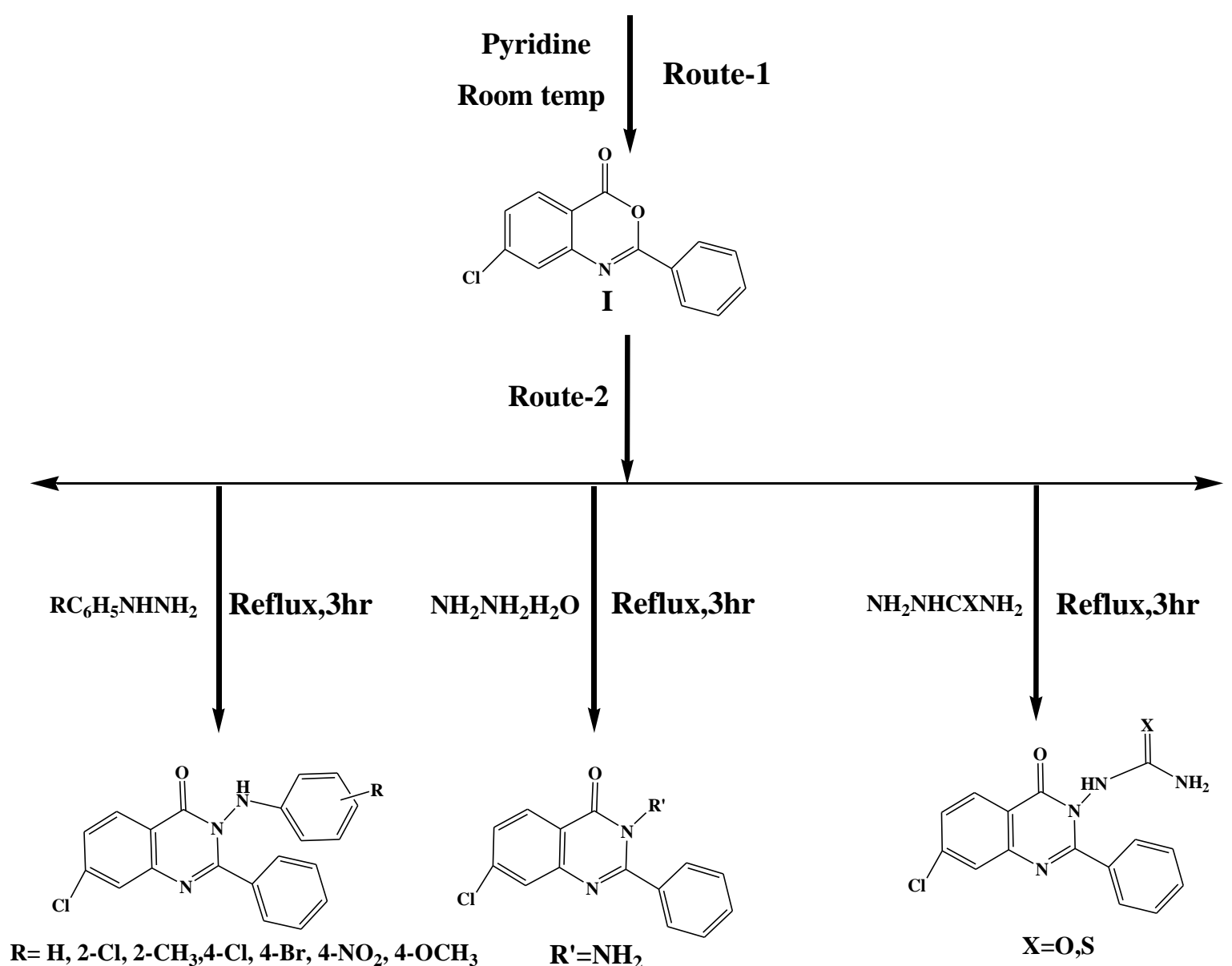

\section{IIa-IIg}

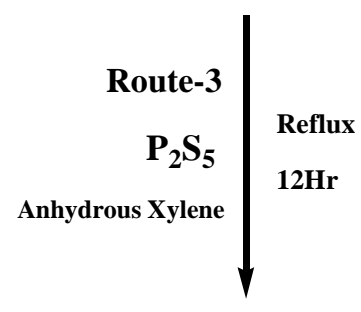<smiles>[R]c1ccc(Nn2c(-c3ccccc3)nc3cc(Cl)ccc3c2=S)cc1</smiles>

$\mathrm{R}=\mathrm{H}, 2-\mathrm{Cl}, 2-\mathrm{CH}_{3}, 4-\mathrm{Cl}, 4-\mathrm{Br}, 4-\mathrm{NO}_{2}, 4-\mathrm{OCH}_{3}$ IIIa-IIIg
IIh

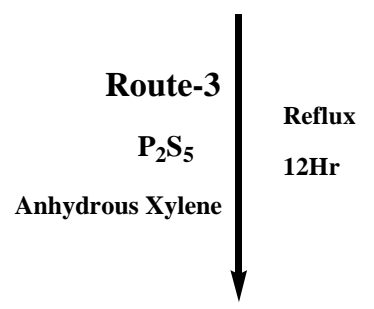

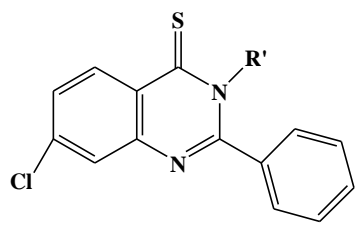

$\mathbf{R}^{\prime}=\mathrm{NH}_{2}$

IIIh
III-IIj

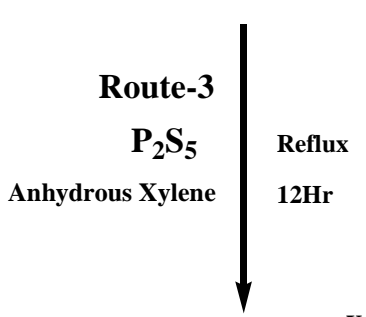<smiles>C=C1c2ccc(Cl)cc2N=C(c2ccccc2)N1NC(C)=O</smiles>

IIIi-IIIj

Fig. 2: Scheme for the synthesis of 7-chloro-3-[substituted (amino/ phenyl amino)]-2-phenyl quinazolin-4 (3H)-one/thione derivatives and 1- (7-chloro-4-oxo/-2phenylquinazoline-3 (4H-yl)) substituted urea 


\section{Pharmacological activity Anti-microbial activity}

All the synthesized compounds were evaluated for their anti-bacterial and anti-fungal activities. A comparative antimicrobial study of the synthesized compounds with that of the standard drugs is effectively presented in table 1 . The present study showed that some of the synthesized quinazoline derivatives performed significant anti-microbial potency. By correlating the structure of the sample compounds with their pharmacological activity, it has been observed that the compounds IIg and IIh showed potent activity against bacteria $S$. aureus, and B. subtilis. This may be due to the presence of electron donating substituents i.e $\mathrm{NH}_{2}$ and p-methoxy phenyl hydrazine group substitution at the $3^{\text {rd }}$ position of quinazoline ring. Similarly compounds like IIc, IIi, IIj also exhibited good activity aganist A.niger and C.albicans respectively due to the aforesaid reason.

The influence of these electron donating groups in improving the antimicrobial activity was revealed through literature (Fu et al., 2010). Compounds containing electron withdrawing groups such as 2-chloro phenyl hydrazine (IIb), 4chloro phenyl hydrazine (IId), p-bromo phenyl hydrazine (IIe), pnitro phenyl hydrazine (IIf) did not exhibit significant antimicrobial activity. Compounds IIIc, IIIg, IIIh, IIIi, IIIj showed mild to moderate anti-microbial activity.

These substitutions are quiet similar to the structure i.e. IIc, IIg, IIh, III, IIj respectively. But there is a substitution at $4^{\text {th }}$ position with sulphur instead of oxygen in the quinazoline ring. So the alteration of activity may be due to the replacement in the quinazoline scaffold.

\section{Analgesic and anti-inflammatory activity}

In the present study, we have screened the analgesic activity by tail immersion method and anti-inflammatory activity by carageenan induced paw edema method as shown in the table 2 and 3. Among the synthesized compounds, compounds IIc and IIg showed significant analgesic and anti-inflammatory activity as compared to the standard drug diclofenac sodium $(20 \mathrm{mg} / \mathrm{kg})$ respectively. This is assumed to be due to the presence of o-methyl phenyl hydrazine and p-methoxy phenyl hydrazine group at $3^{\text {rd }}$ position of the quinazoline ring respectively. The literature survey showed that the therapeutic potency is due to the presence of alkyl or alkyloxy group (Alagarsamy et al., 2005). Similarly compounds containing an amino group (IIh), semicarbamoyl group (IIi), thiosemicarbamoyl group (IIj) showed good anti-inflammatory activity. The activity is probably due to the presence of electron donating substituents in the quinazoline scaffold as mentioned above (Alafeefy et al., 2010). Compounds (IIIc, IIIg, IIIh, IIIi and IIIj) showed mild to moderate analgesic and antiinflammatory activity.

The process of pain and inflammation includes sensitization of the nociceptors, transmission of signal of pain through impulses to the spinal cord and then the pain centre in the thalamus followed by integration of the sensation in the sensory cortex as pain (Yokota et al., 1989). Thus the anti-nociceptive action of the synthesized compounds may exert action either through peripherally or centrally at the site of the nociceptors. The potent inhibitory effects of the synthesized compounds of tail emersion method induced peripheral or cerebral mechanisms. This can only be pinpointed by investigating the effect of the compounds on the main sensitizers of the peripheral pain receptors mainly the prostaglandins.

The carrageenan induced edema is supposed to be mediated through the release of various pain mediators such as serotonin (5-Hydroxy typtamine), prostaglandin etc (Damas et al., 1999). Hence the experimentally observed anti-inflammatory activities of the compounds were found to be due to the inhibition of these mediators.

\section{Acute toxicity studies}

Toxicological studies of the most promising antimicrobial (IIc, IIg, IIh, IIi and $\mathbf{I I j}$ ) analgesic (IIc and IIg) and anti-inflammatory (IIc, $\mathbf{I I g}, \mathbf{I I h}, \mathbf{I} \mathbf{I i}$ and $\mathbf{I I j}$ ) active agents were performed using $\mathrm{LD}_{50}$ standard method in mice in 500, 750 and $1000 \mathrm{mg} / \mathrm{kg}$ (body weight). However, no toxic symptoms or mortality rates were observed $24 \mathrm{hr}$ post administration implying their good safety margin. But apart from these synthesized compounds rest of the synthesized quinazoline derivatives induced urination while compounds induced calmness, muscle relaxation and vasodilation. However, no toxic symptoms or mortality rates were observed after $24 \mathrm{hr}$.

\section{CONCLUSION}

A new series of quinazoline derivatives with a common skeleton were synthesised and evaluated for their anti-microbial, analgesic and anti-inflammatory principle. In general, electron donating group substituted derivatives exhibited better antimicrobial principle than electron withdrawing compounds. The quinazoline derivatives obtained from this research work indicates that the methyl/methoxy group in phenyl hydrazine ring at $3^{\text {rd }}$ position, amine, urea and thiourea substitution at $3^{\text {rd }}$ position of quinzoline derivatives are essential for anti-microbial, analgesic and anti-inflammatory principle.

Compounds IIc, IIg, IIh, III and IIj were found to be potent compound which may be effective as potential source for the development of anti-microbial, analgesic and antiinflammatory compound having common quinazoline scaffold with lesser side effects. Therefore the study deserves further investigation with respect to in vitro activity of the above potent quinazoline derivatives.

\section{ACKNOWLEDGMENTS}

The authors acknowledged the assistance of SAIF, North-Eastern Hill University, Shillong, Meghalaya for analytical support regarding spectral analysis.

\section{Financial support and sponsorship: Nil.}


Conflict of interests: There are no conflicts of interest.

\section{REFERENCES}

Abdel-Rahman TM. Synthesis of some new biologically active 2, 3-disubstituted quinazolin-4-ones. Heterocycl Commun, 1997; 3: 535544.

Adel S, Azab E, Mohamed A, Omar A, Alaa AM, Naglaa I, Abdel A, Magda A, Sayed E, Abdulaziz MA, Mohamed MS, Sami G. Design, synthesis and biological evaluation of novel quinazoline derivatives as potential antitumor agents: Molecular docking study. Eur J Med Chem, 2010; 45: 4188-4198.

Adel S, El-Azab, Kamal EH ElTahir. Design and synthesis of novel 7-aminoquinazoline derivatives: Antitumor and anticonvulsant activities. Bioorg Med Chem Lett, 2012; 22: 1879-1885.

Alafeefy AM, Kadi AA, Al-Deeb OA, El-Tahir KE, Al-Jaber NA. Synthesis, analgesic and anti-inflammatory evaluation of some novel quinazoline derivatives. Eur J Med Chem, 2010; 45: 4947-4962.

Alagarsamy V, Rajasolomon V, Meena R, Kona VR. Synthesis, analgesic, anti-inflammatory and antibacterial activities of some novel 2Butyl-3-substituted quinazolin-4-(3H)-ones. Biol Pharm Bull, 2005; 28 : 1091-1094.

Bekhit AA, Fahmy HT. Design and synthesis of some substituted 1H-pyrazolyl-oxazolidines or $1 \mathrm{H}$-pyrazolyl-thiazolidines as anti-inflammatory and anti-microbial agents. Arch Pharm Pharm Med Chem (Weinheim), 2013; 336, 111-118.

Chandrika PM, Rao ARR, Narsaiah B, Narsaiah MB. Quinazoline derivatives with potent anti-inflammatory and anti-allergic activities. Int J Chem Sci, 2008; 6: 1119-1146.

Chavan BB, Bhalawane PP, Kolsure AK, Chabukswar AR. Synthesis and evaluation of some new 4, 6-disubstituted quinazoline derivatives for antimicrobial and antifungal activities. Asian J Biomed Pharm Sci, 2014; 4: 43-46.

Damas J, Liegeois J, Schmiedebergs FN. The inflammatory reaction induced by formalin in the rat paws Arch Pharmacol, 1999; 359: 220-227.

El-Gazzar ABA, Youssef MM, Youssef AMS, Abu-Hashem AA, Badria FA. Design and synthesis of azolopyrimidoquinolines, pyrimidoquinazolines as anti-oxidant, anti-inflammatory and analgesic activities. Eur J Med Chem, 2009; 44: 609-624.

Fu J, Cheng K, Zhang ZM, Fang RQ, Zhu HL. Synthesis, structure and structure-activity relationship analysis of caffeic acid amides as potential antimicrobials. Eur J Med Chem, 2010; 45: 2638-2643.

Hassan HY, Ismaiel AA, El-Sherief HAH. Studies on synthesis of some quinazolinones bearing different heterocyclic moieties with expected biological activity. Eur J Med Chem, 1991; 26: 743-748.

Hemlatha K, Girija K. Synthesis of some novel 2, 3disubstituted quinazolinone derivatives as analgesic and anti-inflammatory agents. Int J Pharm Pharm Sci, 2011; 3: 103-106.

Ilangovan PS, Ganguly S, Pandit J. Design and synthesis of novel quinazolinone derivatives as broad spectrum anticonvulsant and antimicrobial agent. J Pharm Res, 2010; 3: 703-706.

Jatav V, Mishra P, Kashaw S, Stables,JP. Synthesis and CNS depressant activity of some novel 3-[5-substituted 1, 3, 4-thiadiazole-2-yl]2-styryl quinazoline-4(3H)-ones. Eur J Med. Chem, 2008; 43:135-141.
Jatav V, Mishra P, Kashaw S, Stables JP. CNS depressant and anticonvulsant activities of some novel 3-[5-substituted 1,3,4-thiadiazole2-yl]-2-styryl quinazoline-4(3H)-ones. Eur J Med Chem, 2008; 43: 19451954.

Jiang S, Zeng Q, Gettayacamin M, Tungtaeng A, Wannaying S, Lim A, Hansukjariya P, Okunji CO, Zhu S, Fang D. Antimalarial activities and therapeutic properties of febrifugine analogs. Antimicrob Agents Chemother, 2005; 49: 1169-1176.

Kashaw SK, Kashaw V, Mishra P, Jain NK. Design, synthesis and potential CNS activity of some novel 1-(4-substituted phenyl)-3-(4oxo-2-propyl-4H-quinazolin-3-yl)-urea. Arkivoc, 2008; Xiv: 17-26.

Kashaw SK, Kashaw V, Mishra P, Jain NK, Stables JP. Synthesis, anticonvulsant and CNS depressant activity of some new bioactive 1-(4-substituted-phenyl)-3-(4-oxo-2-phenyl/ethyl-4Hquinazolin-3-yl)-urea. Eur J Med Chem, 2009; 44: 4335-4343.

Lokhandwala S, Patel D. Pharmcological activities of some 4Chloro quinazolinone derivatives. Int J Pharm Tech Res, 2013; 5: 11261131.

Maddeshiya JP, Agarwal S. Synthesis, characterization and antimicrobial activity of some newer quinazoline derivatives. Int J Pharm Erudition, 2013; 2: 1-11.

Mosaad SM, Mohammed KI, Ahmed MA, Abdel-Hamide SG. Synthesis of Certain New 6-Iodoquinazolines as potential antitubercular agents. J Appl Sci, 2004; 4: 302-307.

Patel NB, Patel JC. Synthesis and antimicrobial activity of schiff bases and 2-azetidinones derived from quinazolin-4(3H)-one. Arabian J Chem, 2011; 4: 403-411.

Sarvanan G, Algarsamy V, Prakash CR. Design, synthesis and anticonvulsant activities of novel 1-(substituted/unsubstituted benzylidene)-4-(4-(6,8-dibromo-2-(methyl/phenyl)-4-oxoquinazolin-

3(4H)-yl)phenyl) semicarbazide derivatives. Bioorg Med Chem Lett, 2012; 22: 3072-3078.

Sarvanan G, Algarsamy V, Prakash CR. Synthesis, characterization and in vitro antimicrobial activity of some 1(substitutedbenzylidene)-4-(4-(2-(methyl/phenyl)-4-oxoquinazolin-3(4H)yl)phenyl) semicarbazide derivatives. J. Saudi Chem Soc, 2015; 19: 3-11.

Srivastava AVK, Kumar A. Synthesis of some newer derivatives of substituted quinazolinonyl-2-oxo/thiobarbituric acid as potent anticonvulsant agents. Bioorg Med Chem Lett, 2004; 12: 12571264.

Yokota T. Thalamic mechanism of pain: Shell theory of thalamic nociception. Jpn J Physiol 1989; 39:335-348.

\section{How to cite this article:}

Dash B, Dash S, Laloo D, Medhi C. Design, Synthesis and Preliminary Pharmacological Screening (antimicrobial, analgesic and anti-inflammatory activity) of Some Novel Quinazoline Derivatives. J App Pharm Sci, 2017; 7 (06): 083-096. 